\title{
Symmetrical trimeric star-shaped mesogens based on 1,3,5-trisubstituted benzene incorporating Schiff base and azobenzene fragments as the peripheral units: Synthesis and mesomorphic properties
}

\author{
GUAN-YEOW YEAP ${ }^{\mathrm{a}, *}$, YEW-HONG OOI ${ }^{\mathrm{a}}$, NOZOMI UCHIDA ${ }^{\mathrm{b}}$ and MASATO M ITO $^{\mathrm{b}}$ \\ ${ }^{a}$ Liquid Crystal Research Laboratory, School of Chemical Sciences, Universiti Sains Malaysia, \\ 11800 Minden, Penang, Malaysia \\ ${ }^{b}$ Department of Environmental Engineering for Symbiosis, Faculty of Engineering, Soka University, \\ Hachioji, Tokyo 192-8577, Japan \\ e-mail: gyyeap@usm.my
}

MS received 27 July 2013; revised 2 December 2013; accepted 30 December 2013

\begin{abstract}
Two series of symmetrical three-armed star-shaped mesogens based on 1,3,5-trihydroxybenzene as a core unit, interconnecting three Schiff base or azobenzene moieties via oxymethylene spacers have been synthesized and characterized by spectroscopic techniques. Every member in these series possesses either chlorine $(\mathrm{Cl})$ or bromine $(\mathrm{Br})$ terminal atom, with different alkyl spacer length $\left(\mathrm{C}_{n} \mathrm{H}_{2 n}\right.$ whereby $n$ ranging from 3 to 6). Their thermal stability and mesomorphic properties are investigated by employing DSC and POM. The dependence of phase transition in relation to the alkyl spacer length is shown by both series. These star-shaped mesogens exhibit only nematic and smectic phases. The difference between the two series lies on the structure of linking group in the peripheral units ( $-\mathrm{CH}=\mathrm{N}$ - for series PSB-X-n and $-\mathrm{N}=\mathrm{N}$ - for series PAZ-X-n). Therefore, a comparison study of the mesomorphic properties between these two series of star-shaped mesogens is discussed whereby the azobenzene-based mesogens are thermally more stable than the Schiff base counterpart. In addition, soft crystalline phase is observed for the azobenzene-based star-shaped mesogens possessing hexyl alkyl spacer.
\end{abstract}

Keywords. Star-shaped liquid crystals; symmetrical; terminal substituent; Schiff base and azobenzene moieties; comparative study.

\section{Introduction}

In the last two decades, the development of non-conventional liquid crystals has received overwhelming interest from researchers due to its great potential to serve as novel medium in electro-optical applications, sensing applications and electron/ion/molecular transporting system. ${ }^{1,2}$ Generally, the prototype of conventional liquid crystal molecule consists of a rodlike rigid core or a relatively flat disc-shaped core substituted by terminal flexible chain and small lateral substituent. In contrast, non-conventional LCs are molecules with an anisotropic shape that deviates from the classical rod- and disc-shaped molecules. ${ }^{3}$ They are made up of complex self-organising molecules comprising discrete molecular fragment namely flexible

\footnotetext{
*For correspondence
}

components (tails and spacers) and several anisometric (mesogenic) segments which are chemically incompatible with each other. ${ }^{1,4,5}$ On account of their astonishing self-assembly into complex soft materials, nonconventional LCs are of massive interest at present. To date, there are various types of non-conventional LCs documented in the literature (example, such as multiarm mesogens,${ }^{6-8}$ oligomers, ${ }^{9-13}$ bent-core or V-shaped mesogens, ${ }^{14-18}$ metallomesogens ${ }^{19-22}$ and hydrogenbonded mesogens). ${ }^{23-25}$ Thus, the interesting properties of these materials have prompted researchers to further study and investigate novel non-conventional materials which can eventually lead to new findings on novel mesophase morphologies and deeper insights in the driving force of their self-organization.

Among the various types of non-conventional LCs, star-shaped mesogens is considered as one of the most popular studied materials in recent year. The simplest star-shaped mesogen is termed as Hekates, ${ }^{26,27}$ which consists of only three identical mesogenic/ non-mesogenic groups symmetrically attached to a 
disc-shaped core unit, hence representing yet another important class of low molecular mass liquid crystals. Star-shaped LCs are attractive because of their ability to exhibit unique optical behaviour and a broad range of mesophases such as soft crystalline phases, nematic, smectic, cholesteric, as well as columnar phases. ${ }^{28-33}$ The driving force for the type of mesophase formed is presumably dominated by the peripheral units. Nevertheless, the length of spacer also plays a crucial role in the mesophase stability. For instance, star-shaped mesogens possessing short spacer hardly show any mesophase as the short chain may prevent the peripheral units from the appropriate arrangement in the liquid crystalline phase. However, when the flexible spacer length is long enough, the peripheral units are capable of folding an anisotropic shape to give rise to the appearance of liquid crystalline state. A related example can be shown by the phloroglucinol-based star-shaped mesogens whereby homologues with butyl spacer or higher $(n \geq 4)$ exhibit cholesteric phase and the mesophase interval of the cholesteric phase widens significantly. ${ }^{28}$ Besides, star-shaped LCs comprised of a benzene core unit with three extended peripheral unit connected via flexible spacers can form low temperature glassy state. ${ }^{29}$ Hence, they own great potential to be utilized as soft functional and glass-forming liquid crystals materials. This type of low molar mass compound is most preferred over polymeric glass-forming LCs due to their lower viscosity which can eventually provide fast and better orientation. ${ }^{34}$ In addition, glass-forming LCs also possess many special optical, mechanical and thermal stable properties. ${ }^{29}$ Thus, star-shaped LCs are especially important not only for the theoretical understanding of liquid crystalline phenomenon, but also attractive in the view of preparing novel functional materials owing to their unique physical and optical properties.

Our present interest focuses on the investigation of phloroglucinol-based star-shaped mesogens incorporating Schiff base and azobenzene moieties as the peripheral units. The intermediary Schiff base or azobenzene compounds possessing chloro $(\mathrm{Cl})$ or bromo $(\mathrm{Br})$ as the terminal substituent with varying spacer length $(n)$ are joined to the 1,3,5-position of phloroglucinol to yield the symmetrical three-armed star-shaped molecules. The acronyms used to refer the two series of star-shaped mesogens in this article are PSB-X- $\boldsymbol{n}$ and PAZ-X- $\boldsymbol{n}$, in which PSB-X- $\boldsymbol{n}$ is referred to the star-shaped mesogen incorporating Schiff base moieties while PAZ-X- $\boldsymbol{n}$ is referred to the mesogen comprising azobenzene fragments as the peripheral units. The symbol $\mathbf{X}$ denotes the terminal chloro or bromo substituent, while $\boldsymbol{n}$ denotes the number of methylene units linking the peripheral

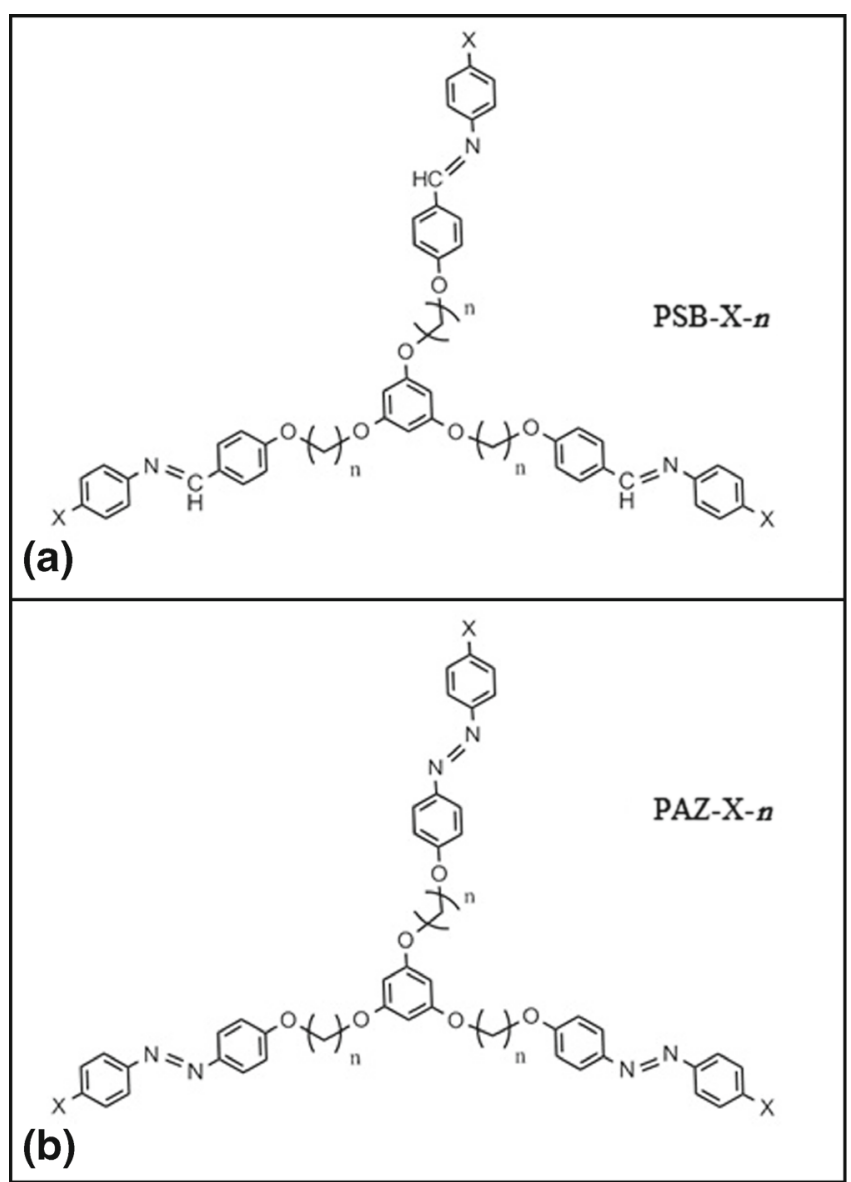

Figure 1. Molecular structure of the symmetrical trimeric star-shaped mesogens (a) PSB-X- $\boldsymbol{n}$ and (b) PAZ-X- $\boldsymbol{n}$ (where $\mathrm{X}=\mathrm{Cl}, \mathrm{Br}$ and $\mathrm{C}_{n} \mathrm{H}_{2 n}$ where $n=3,4,5,6$ ).

unit and the benzene core. The molecular structure of present star-shaped mesogens are depicted in figure 1. Since the molecular structure of the star-shaped compounds and their liquid crystalline properties are closely related, therefore, the structure-mesomorphic property relationship in terms of the linking group, terminal substituent and spacer length towards the mesomorphic properties of the present star-shaped liquid crystals are described.

\section{Experimental}

\subsection{Chemicals and reagents}

4-Chloroaniline, 4-bromoaniline, phenol, 1,3-dibromopropane, 1,4-dibromobutane, 1,5-dibromopentane and 1,6-dibromohexane were obtained from Merck (Germany). Phloroglucinol anhydrous and 4-hydroxybenzaldehyde were purchased from Acros (Belgium). Potassium carbonate anhydrous and sodium 
nitrate were purchased from R\&M Chemicals (UK) while potassium iodide was obtained from Fisher Scientific (USA). All chemicals and solvents were used directly without any purification.

\subsection{Methods and characterization}

The molecular structure of all the star-shaped compounds and their intermediates were confirmed and substantiated by ${ }^{1} \mathrm{H}$ and ${ }^{13} \mathrm{C}$-NMR spectroscopy along with two-dimensional NMR experiments $\left({ }^{1} \mathrm{H}-{ }^{1} \mathrm{H}\right.$ COSY, ${ }^{1} \mathrm{H}_{-}{ }^{13} \mathrm{C}$ HMQC and ${ }^{1} \mathrm{H}_{-}{ }^{13} \mathrm{C}$ HMBC), using a Bruker Avance $500 \mathrm{MHz}$ spectrometer. Deuterated chloroform $\left(\mathrm{CDCl}_{3}\right)$ was used as the solvent with tetramethylsilane (TMS) as internal standard. Elemental analysis of the final products in terms of carbon, hydrogen and nitrogen were measured using Perkin Elmer 2400 LS series CHSN/O analyzer. While the FT-IR spectra were recorded using Perkin Elmer 2000-FTIR spectrophotometer in a frequency range of $4000-400 \mathrm{~cm}^{-1}$ in which the samples were embedded in $\mathrm{KBr}$ discs. The thermal behaviour of the star-shaped mesogens were measured through differential scanning calorimetry (DSC) using a Seiko DSC120 Model 5500 differential scanning calorimeter. The scanning profile in all cases was heated and cooled at the rate of $\pm 5^{\circ} \mathrm{C}$. Texture characterization was performed using a Carl Zeiss Axioskop 40 Polarizing Optical Microscope equipped with a Linkam LTS350 hot stage. The samples were prepared as a thin film sandwiched between a glass slide and a cover.

\subsection{Synthesis}

The synthetic pathway towards the formation of the star-shaped mesogens is outlined in scheme 1. The intermediates SB-X-n were prepared by using the procedure as reported by Yeap et al.,${ }^{35}$ while AZ-X-n were prepared according to the method described by García et $a l .{ }^{36}$ The preparation of the star-shaped compounds, PSB-X- $\boldsymbol{n}$ and PAZ-X- $\boldsymbol{n}$ will be described in the following section. Overall, the representative codes for PSB-X- $\boldsymbol{n}$ and PAZ-X- $\boldsymbol{n}$ with respective terminal atom, linking group and spacer were summarized in table 1 .
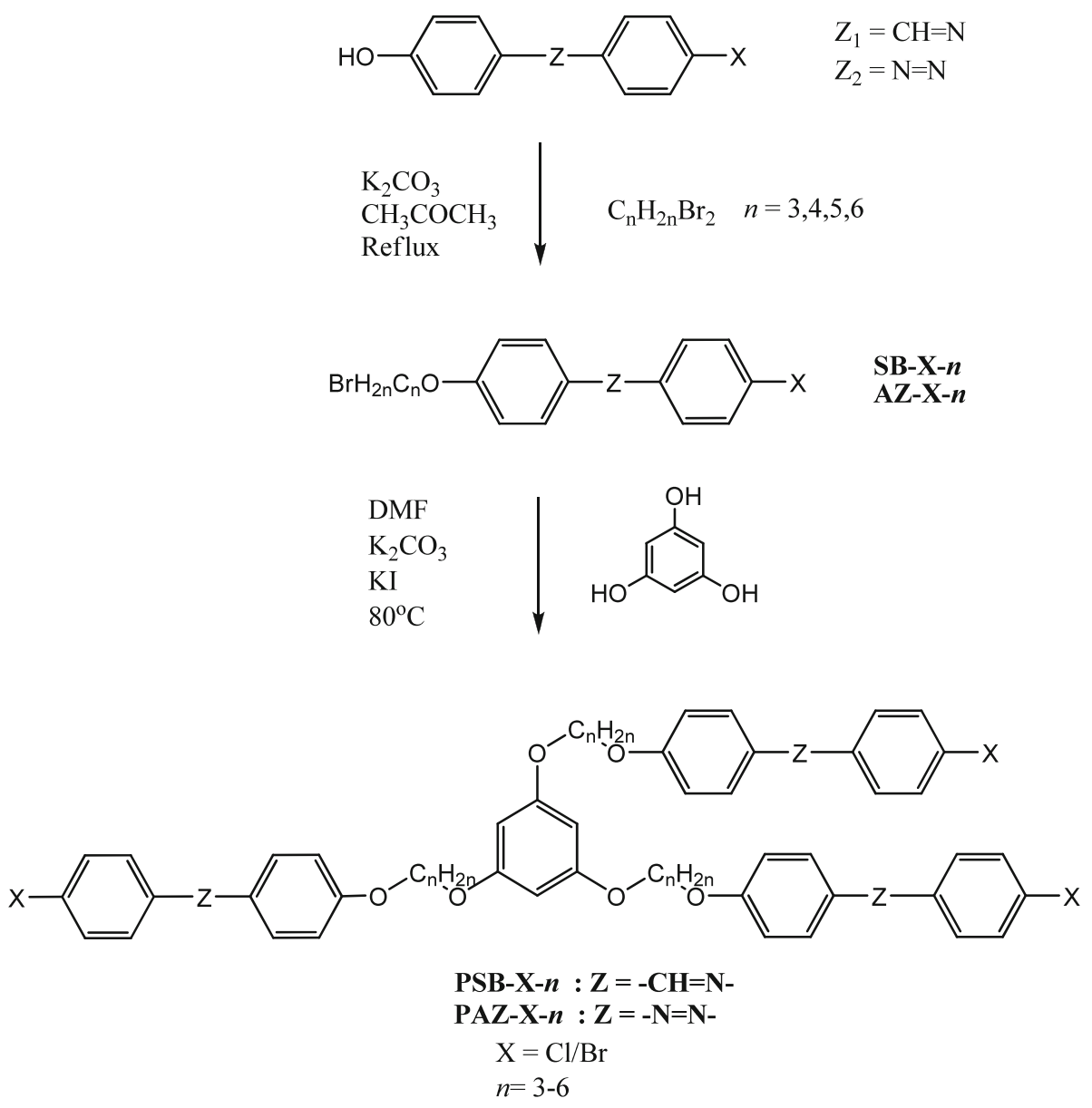

Scheme 1. Synthetic route towards the preparation of three-armed star-shaped mesogens PSB-X-n and PAZ-X-n. 
Table 1. The linking group (Z), terminal substituent (X) and the spacer length $(n)$ of the star-shaped mesogen, PSB-X- $\boldsymbol{n}$ and PAZ-X- $\boldsymbol{n}$.

Terminal

Compound Linking group, $\mathrm{Z} \quad$ substituent, $\mathrm{X} \quad$ Spacer, $n$

\begin{tabular}{llll}
\hline PSB-CL-3 & $\mathrm{CH}=\mathrm{N}$ & $\mathrm{Cl}$ & 3 \\
PSB-CL-4 & $\mathrm{CH}=\mathrm{N}$ & $\mathrm{Cl}$ & 4 \\
PSB-CL-5 & $\mathrm{CH}=\mathrm{N}$ & $\mathrm{Cl}$ & 5 \\
PSB-CL-6 & $\mathrm{CH}=\mathrm{N}$ & $\mathrm{Cl}$ & 6 \\
PSB-BR-3 & $\mathrm{CH}=\mathrm{N}$ & $\mathrm{Br}$ & 3 \\
PSB-BR-4 & $\mathrm{CH}=\mathrm{N}$ & $\mathrm{Br}$ & 4 \\
PSB-BR-5 & $\mathrm{CH}=\mathrm{N}$ & $\mathrm{Br}$ & 5 \\
PSB-BR-6 & $\mathrm{CH}=\mathrm{N}$ & $\mathrm{Br}$ & 6 \\
PAZ-CL-3 & $\mathrm{N}=\mathrm{N}$ & $\mathrm{Cl}$ & 3 \\
PAZ-CL-4 & $\mathrm{N}=\mathrm{N}$ & $\mathrm{Cl}$ & 4 \\
PAZ-CL-5 & $\mathrm{N}=\mathrm{N}$ & $\mathrm{Cl}$ & 5 \\
PAZ-CL-6 & $\mathrm{N}=\mathrm{N}$ & $\mathrm{Cl}$ & 6 \\
PAZ-BR-3 & $\mathrm{N}=\mathrm{N}$ & $\mathrm{Br}$ & 3 \\
PAZ-BR-4 & $\mathrm{N}=\mathrm{N}$ & $\mathrm{Br}$ & 4 \\
PAZ-BR-5 & $\mathrm{N}=\mathrm{N}$ & $\mathrm{Br}$ & 5 \\
PAZ-BR-6 & $\mathrm{N}=\mathrm{N}$ & $\mathrm{Br}$ & 6 \\
\hline
\end{tabular}

\subsection{Synthesis of 4-[(4-substituted-}

phenylimino)methyl]phenoxy-n-bromoalkane,

\section{$\boldsymbol{S B}-\boldsymbol{X}-\boldsymbol{n}$}

4-[(4-Chlorophenylimino)methyl]phenol or 4-[(4-bromophenylimino)methyl]phenol was synthesized via condensation reaction in methanol between 4-hydroxybenzaldehyde and 4-chloro- or 4-bromoaniline. The Schiff base compounds were further reacted with various $\alpha, \omega$-dibromoalkanes ranging from $\mathrm{C}_{3} \mathrm{H}_{6} \mathrm{Br}_{2}$ to $\mathrm{C}_{6} \mathrm{H}_{12} \mathrm{Br}_{2}$ through Williamson etherification to afford the $\omega$-brominated intermediary Schiff base side-chain,

SB-X-n.

\subsection{Synthesis of 4-(4-substituted-}

phenyldiazenyl)phenoxy-n-bromoalkane,

\section{AZ-X-n}

Azobenzene compounds 4-[(4-chlorophenyl)diazenyl] phenol or 4-[(4-bromophenyl)diazenyl]phenol were prepared through diazonium coupling between the diazonium ion (obtained from treating the substitutedaniline with diluted hydrochloric acid and sodium nitrate in $0-5^{\circ} \mathrm{C}$ ) and phenol. The azobenzene compounds were further alkylated via Williamson etherification with various $\alpha, \omega$-dibromoalkanes ranging from $\mathrm{C}_{3} \mathrm{H}_{6} \mathrm{Br}_{2}$ to $\mathrm{C}_{6} \mathrm{H}_{12} \mathrm{Br}_{2}$ in order to yield the $\omega$-brominated intermediary azo side-chain, $\mathbf{A Z - X}-\boldsymbol{n}$.
2.6 Synthesis of 1,3,5-tris\{4-[(4-substitutedphenylimino)methyl]phenoxy\}-n-alkyloxy benzene, PSB- $X-n(X=C l, B r ; n=3,4,5,6)$

Phloroglucinol anhydrous $(0.31 \mathrm{~g}, 2.5 \mathrm{mmol})$ was first dissolved in $10 \mathrm{~mL}$ of acetone. Potassium carbonate anhydrous $(7.00 \mathrm{~g}, 50 \mathrm{mmol})$ was added to the solution and allowed to stir at reflux for $1 \mathrm{~h}$. Excess amount of compound SB-X-n (10 mmol) was dissolved in $50 \mathrm{~mL}$ of hot $\mathrm{N}, \mathrm{N}^{\prime}$-dimethylformamide (DMF) and was added to the reaction mixture followed by a catalytic amount of potassium iodide. The reaction mixture was stirred at $80^{\circ} \mathrm{C}$ for $48 \mathrm{~h}$ (2 days). Then, it was allowed to cool down and left to evaporate at room temperature until the total volume of the mixture was $40 \mathrm{~mL}$. Cold water was added and the resulting precipitate was filtered off, dried and purified by column chromatography whereby dichloromethane was used as the mobile phase. The precipitate thus obtained was recrystallized from acetone to obtain the star-shaped compounds, PSB-X-n. The physical data, IR, ${ }^{1} \mathrm{H}$ - and ${ }^{13} \mathrm{C}-\mathrm{NMR}$ along with elemental results were listed as follows:

PSB-CL-3: Yield: 43\% White precipitate. Elemental analysis: found, $\mathrm{C}$ 69.02, H 5.30, N 4.51; calculated $\left(\mathrm{C}_{54} \mathrm{H}_{48} \mathrm{~N}_{3} \mathrm{O}_{6} \mathrm{Cl}_{3}\right), \mathrm{C}$ 68.90, H 5.14, N 4.46. IR ( KBr) $v / \mathrm{cm}^{-1}: 2946,2870$ (C-H aliphatic), $1619(\mathrm{C}=\mathrm{N}), 1571$, 1602 (C=C), 1250 (C-O ether). ${ }^{1} \mathrm{H}-\mathrm{NMR}\left(\mathrm{CDCl}_{3}\right)$

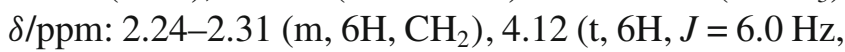
$\left.\mathrm{OCH}_{2}\right), 4.20\left(\mathrm{t}, 6 \mathrm{H}, J=6.0 \mathrm{~Hz}, \mathrm{OCH}_{2}\right), 6.12(\mathrm{~s}, 3 \mathrm{H}$, Ar), $6.97(\mathrm{~d}, 6 \mathrm{H}, J=9.0 \mathrm{~Hz}, \operatorname{Ar}), 7.11(\mathrm{~d}, 6 \mathrm{H}, J=$ $8.5 \mathrm{~Hz}, \mathrm{Ar}), 7.33$ (d, 6H, $J=8.5 \mathrm{~Hz}, \mathrm{Ar}), 7.81$ (d, $2 \mathrm{H}, J=8.5 \mathrm{~Hz}, \mathrm{Ar}$ ), 8.33 (s $3 \mathrm{H}, \mathrm{CH}=\mathrm{N}$ ). ${ }^{13} \mathrm{C}-\mathrm{NMR}$ $\left(\mathrm{CDCl}_{3}\right) \delta / \mathrm{ppm}: 29.20\left(\mathrm{C}_{\text {aliphatic }}\right), 64.39,64.65\left(\mathrm{C}_{\text {arom }}{ }^{-}\right.$ $\left.\mathrm{OCH}_{2}\right), 94.23,114.77,122.19,129.09,129.19,130.62$ $\left(\mathrm{C}_{\text {arom }}\right), 131.06\left(\mathrm{C}_{\text {arom }}-\mathrm{Cl}\right), 150.80\left(\mathrm{C}_{\text {arom }}-\mathrm{N}\right), 159.93$ $(\mathrm{C}=\mathrm{N}), 160.75,161.73\left(\mathrm{C}_{\text {arom }}-\mathrm{O}\right)$.

PSB-CL-4: Yield: $45 \%$ White precipitate. Elemental analysis: found, C 69.77, H 5.64, N 4.21; calculated $\left(\mathrm{C}_{57} \mathrm{H}_{54} \mathrm{~N}_{3} \mathrm{O}_{6} \mathrm{Cl}_{3}\right), \mathrm{C}$ 69.62, H 5.53, N 4.27. IR (KBr) $v / \mathrm{cm}^{-1}: 2947,2874$ (C-H aliphatic), $1620(\mathrm{C}=\mathrm{N}), 1573$, $1604(\mathrm{C}=\mathrm{C}), 1250$ (C-O ether). ${ }^{1} \mathrm{H}-\mathrm{NMR}\left(\mathrm{CDCl}_{3}\right)$

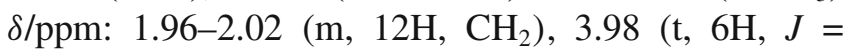
$\left.5.7 \mathrm{~Hz}, \mathrm{OCH}_{2}\right), 4.07\left(\mathrm{t}, 6 \mathrm{H}, J=5.7 \mathrm{~Hz}, \mathrm{OCH}_{2}\right), 6.06$ (s, 3H, Ar), 6.95 (d, 6H, $J=8.7 \mathrm{~Hz}, A r), 7.12(\mathrm{~d}, 6 \mathrm{H}$, $J=8.7 \mathrm{~Hz}, \mathrm{Ar}), 7.31$ (d, 6H, $J=8.7 \mathrm{~Hz}, \mathrm{Ar}), 7.82(\mathrm{~d}$, $6 \mathrm{H}, J=8.4 \mathrm{~Hz}, \mathrm{Ar}), 8.32(\mathrm{~s}, 3 \mathrm{H}, \mathrm{CH}=\mathrm{N}) .{ }^{13} \mathrm{C}-\mathrm{NMR}$

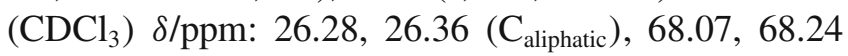
$\left(\mathrm{C}_{\text {arom }}-\mathrm{OCH}_{2}\right), 94.31,115.15,122.60,129.51,129.62$, $131.23\left(\mathrm{C}_{\text {arom }}\right), 132.40\left(\mathrm{C}_{\text {arom }}-\mathrm{Cl}\right), 145.32\left(\mathrm{C}_{\text {arom }}-\mathrm{N}\right)$, $160.41(\mathrm{C}=\mathrm{N}), 161.23,162.44\left(\mathrm{C}_{\text {arom }}-\mathrm{O}\right)$. 
PSB-CL-5: Yield: 42\% White precipitate. Elemental analysis: found, C 70.44, H 5.87, N 4.05; calculated $\left(\mathrm{C}_{60} \mathrm{H}_{60} \mathrm{~N}_{3} \mathrm{O}_{6} \mathrm{Cl}_{3}\right), \mathrm{C} 70.27, \mathrm{H}$ 5.90, N 4.10. IR (KBr) $v / \mathrm{cm}^{-1}$ : 2946, 2869 (C-H aliphatic), $1619(\mathrm{C}=\mathrm{N}), 1571$, 1603 (C=C), 1250 (C-O ether). ${ }^{1} \mathrm{H}-\mathrm{NMR}\left(\mathrm{CDCl}_{3}\right)$ S/ppm: $1.65-1.72\left(\mathrm{~m}, 6 \mathrm{H}, \mathrm{CH}_{2}\right), 1.83-1.92(\mathrm{~m}, 12 \mathrm{H}$, $\left.\mathrm{CH}_{2}\right), 3.97\left(\mathrm{t}, 6 \mathrm{H}, J=6.0 \mathrm{~Hz}, \mathrm{OCH}_{2}\right), 4.07(\mathrm{t}, 6 \mathrm{H}, J$ $\left.=6.0 \mathrm{~Hz}, \mathrm{OCH}_{2}\right), 6.10(\mathrm{~s}, 3 \mathrm{H}, \mathrm{Ar}), 6.90(\mathrm{~d}, 6 \mathrm{H}, J=$ $8.7 \mathrm{~Hz}, \mathrm{Ar}), 7.17$ (d, 6H, $J=8.4 \mathrm{~Hz}, \mathrm{Ar}), 7.36$ (d, 6H, $J$ $=8.7 \mathrm{~Hz}, \mathrm{Ar}), 7.87(\mathrm{~d}, 6 \mathrm{H}, J=8.4 \mathrm{~Hz}, \mathrm{Ar}), 8.37(\mathrm{~s}, 3 \mathrm{H}$, $\mathrm{CH}=\mathrm{N}) .{ }^{13} \mathrm{C}-\mathrm{NMR}\left(\mathrm{CDCl}_{3}\right)$ $\delta / \mathrm{ppm}: 22.72,28.82,28.98$ $\left(\mathrm{C}_{\text {aliphatic }}\right), 67.70,68.18\left(\mathrm{C}_{\text {arom }}-\mathrm{OCH}_{2}\right), 93.23,114.76$, $122.21,129.12,129.27,131.20\left(\mathrm{C}_{\text {arom }}\right), 131.99\left(\mathrm{C}_{\text {arom }}{ }^{-}\right.$ $\mathrm{Cl}), 144.92\left(\mathrm{C}_{\text {arom }}-\mathrm{N}\right), 160.08(\mathrm{C}=\mathrm{N}), 160.90,161.14$ $\left(\mathrm{C}_{\text {arom }}-\mathrm{O}\right)$.

PSB-CL-6: Yield: 41\% Beige precipitate. Elemental analysis: found, $\mathrm{C} 71.05, \mathrm{H} 6.58, \mathrm{~N}$ 4.02; calculated $\left(\mathrm{C}_{63} \mathrm{H}_{66} \mathrm{~N}_{3} \mathrm{O}_{6} \mathrm{Cl}_{3}\right), \mathrm{C} 70.88, \mathrm{H} 6.23, \mathrm{~N}$ 3.94. IR (KBr) $v / \mathrm{cm}^{-1}: 2939,2858$ (C-H aliphatic), $1620(\mathrm{C}=\mathrm{N}), 1573$, $1604(\mathrm{C}=\mathrm{C}), 1251$ (C-O ether). ${ }^{1} \mathrm{H}-\mathrm{NMR}\left(\mathrm{CDCl}_{3}\right)$

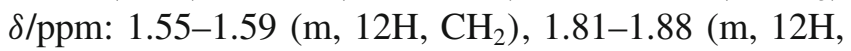
$\left.\mathrm{CH}_{2}\right), 3.95\left(\mathrm{t}, 6 \mathrm{H}, J=6.3 \mathrm{~Hz}, \mathrm{OCH}_{2}\right), 4.06(\mathrm{t}, 6 \mathrm{H}, J$ $\left.=6.3 \mathrm{~Hz}, \mathrm{OCH}_{2}\right), 6.10(\mathrm{~s}, 3 \mathrm{H}, \mathrm{Ar}), 6.99(\mathrm{~d}, 6 \mathrm{H}, J=$ $8.7 \mathrm{~Hz}, \mathrm{Ar}), 7.15(\mathrm{~d}, 6 \mathrm{H}, J=8.7 \mathrm{~Hz}, \mathrm{Ar}), 7.35$ (d, 6H, $J$ $=8.7 \mathrm{~Hz}, \mathrm{Ar}), 7.85(\mathrm{~d}, 6 \mathrm{H}, J=8.7 \mathrm{~Hz}, \mathrm{Ar}), 8.36(\mathrm{~s}, 3 \mathrm{H}$, $\mathrm{CH}=\mathrm{N}) .{ }^{13} \mathrm{C}-\mathrm{NMR}\left(\mathrm{CDCl}_{3}\right) \delta / \mathrm{ppm}: 26.22,26.29,29.50$, $29.57\left(\mathrm{C}_{\text {aliphatic }}\right), 68.21,68.46\left(\mathrm{C}_{\text {arom }}-\mathrm{OCH}_{2}\right), 94.26$, $115.15,123.53,129.58,131.07\left(\mathrm{C}_{\text {arom }}\right), 131.42\left(\mathrm{C}_{\text {arom }}{ }^{-}\right.$ $\mathrm{Cl}), 151.14\left(\mathrm{C}_{\text {arom }}-\mathrm{N}\right), 160.40(\mathrm{C}=\mathrm{N}), 161.35,162.46$ $\left(\mathrm{C}_{\text {arom }}-\mathrm{O}\right)$.

PSB-BR-3: Yield: 38\% Beige precipitate. Elemental analysis: found, $\mathrm{C} 60.52, \mathrm{H} 4.61, \mathrm{~N} 3.95$; calculated $\left(\mathrm{C}_{54} \mathrm{H}_{48} \mathrm{~N}_{3} \mathrm{O}_{6} \mathrm{Br}_{3}\right), \mathrm{C} 60.35, \mathrm{H} 4.50, \mathrm{~N}$ 3.91. IR (KBr) $v / \mathrm{cm}^{-1}$ : 2942, 2868 (C-H aliphatic), $1620(\mathrm{C}=\mathrm{N}), 1571$, 1603 (C=C), 1250 (C-O ether). ${ }^{1} \mathrm{H}-\mathrm{NMR}\left(\mathrm{CDCl}_{3}\right)$

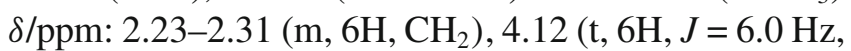
$\left.\mathrm{OCH}_{2}\right), 4.21\left(\mathrm{t}, 6 \mathrm{H}, J=6.0 \mathrm{~Hz}, \mathrm{OCH}_{2}\right), 6.12(\mathrm{~s}, 3 \mathrm{H}$, $\mathrm{Ar}), 6.97(\mathrm{~d}, 6 \mathrm{H}, J=8.7 \mathrm{~Hz}, \operatorname{Ar}), 7.06(\mathrm{~d}, 6 \mathrm{H}, J=$ $8.7 \mathrm{~Hz}, \mathrm{Ar}), 7.48$ (d, 6H, $J=8.4 \mathrm{~Hz}, \mathrm{Ar}), 7.81$ (d, $6 \mathrm{H}, J=8.7 \mathrm{~Hz}, \mathrm{Ar}$ ), $8.33(\mathrm{~s}, 3 \mathrm{H}, \mathrm{CH}=\mathrm{N}) .{ }^{13} \mathrm{C}-\mathrm{NMR}$ $\left(\mathrm{CDCl}_{3}\right) \delta / \mathrm{ppm}: 29.20\left(\mathrm{C}_{\text {aliphatic }}\right), 64.40,64.66\left(\mathrm{C}_{\text {arom }}{ }^{-}\right.$ $\left.\mathrm{OCH}_{2}\right), 94.26,114.78,122.58,129.10,130.64,132.14$ $\left(\mathrm{C}_{\text {arom }}\right), 118.04\left(\mathrm{C}_{\text {arom }}-\mathrm{Br}\right), 151.31\left(\mathrm{C}_{\text {arom }}-\mathrm{N}\right), 159.92$ $(\mathrm{C}=\mathrm{N}), 160.76,161.75\left(\mathrm{C}_{\text {arom }}-\mathrm{O}\right)$.

PSB-BR-4: Yield: 43\% Beige precipitate. Elemental analysis: found, $\mathrm{C} 61.40, \mathrm{H} 4.85, \mathrm{~N} 3.80$; calculated $\left(\mathrm{C}_{57} \mathrm{H}_{54} \mathrm{~N}_{3} \mathrm{O}_{6} \mathrm{Br}_{3}\right), \mathrm{C} 61.30, \mathrm{H} 4.87, \mathrm{~N}$ 3.76. IR (KBr) $v / \mathrm{cm}^{-1}: 2944,2873$ (C-H aliphatic), $1623(\mathrm{C}=\mathrm{N}), 1572$, $1603(\mathrm{C}=\mathrm{C}), 1252$ (C-O ether). ${ }^{1} \mathrm{H}-\mathrm{NMR}\left(\mathrm{CDCl}_{3}\right)$ S/ppm: 1.98-2.03 (m, 12H, $\left.\mathrm{CH}_{2}\right), 3.99(\mathrm{t}, 6 \mathrm{H}, J=$ $6.0 \mathrm{~Hz}, \mathrm{OCH}_{2}$ ), 4.09 (t, $6 \mathrm{H}, J=6.0 \mathrm{~Hz}, \mathrm{OCH}_{2}$ ), 6.08 (s, 3H, Ar), $6.96(\mathrm{~d}, 6 \mathrm{H}, J=8.5 \mathrm{~Hz}, \mathrm{Ar}), 7.06(\mathrm{~d}, 6 \mathrm{H}$,
$J=8.5 \mathrm{~Hz}, \mathrm{Ar}), 7.47(\mathrm{~d}, 6 \mathrm{H}, J=8.5 \mathrm{~Hz}, \mathrm{Ar}), 7.81(\mathrm{~d}$, $6 \mathrm{H}, J=8.5 \mathrm{~Hz}, \mathrm{Ar}), 8.33$ (s, 3H, CH=N). ${ }^{13} \mathrm{C}-\mathrm{NMR}$ $\left(\mathrm{CDCl}_{3}\right) \delta /$ ppm: 25.90, $25.98\left(\mathrm{C}_{\text {aliphatic }}\right), 67.47,67.67$ $\left(\mathrm{C}_{\text {arom }}-\mathrm{OCH}_{2}\right), 94.02,114.76,122.60,128.95,130.65$, $132.14\left(\mathrm{C}_{\text {arom }}\right), 118.86\left(\mathrm{C}_{\text {arom }}-\mathrm{Br}\right), 151.33\left(\mathrm{C}_{\text {arom }}-\mathrm{N}\right)$, $160.00(\mathrm{C}=\mathrm{N}), 160.85,161.91\left(\mathrm{C}_{\text {arom }}-\mathrm{O}\right)$.

PSB-BR-5: Yield: $41 \%$ Beige precipitate. Elemental analysis: found, C 62.10, H 5.31, N 3.66; calculated $\left(\mathrm{C}_{60} \mathrm{H}_{60} \mathrm{~N}_{3} \mathrm{O}_{6} \mathrm{Br}_{3}\right), \mathrm{C} 62.19, \mathrm{H} 5.22, \mathrm{~N}$ 3.63. IR (KBr) $v / \mathrm{cm}^{-1}: 2941,2868$ (C-H aliphatic), $1621(\mathrm{C}=\mathrm{N}), 1572$, $1603(\mathrm{C}=\mathrm{C}), 1251$ (C-O ether). ${ }^{1} \mathrm{H}-\mathrm{NMR}\left(\mathrm{CDCl}_{3}\right)$

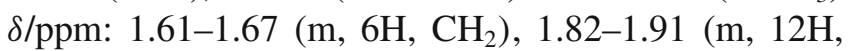
$\left.\mathrm{CH}_{2}\right), 3.95\left(\mathrm{t}, 6 \mathrm{H}, J=6.3 \mathrm{~Hz}, \mathrm{OCH}_{2}\right), 4.05(\mathrm{t}, 6 \mathrm{H}, J$ $\left.=6.3 \mathrm{~Hz}, \mathrm{OCH}_{2}\right), 6.08(\mathrm{~s}, 3 \mathrm{H}, \mathrm{Ar}), 6.96(\mathrm{~d}, 6 \mathrm{H}, J=$ $8.7 \mathrm{~Hz}, \mathrm{Ar}), 7.06(\mathrm{~d}, 6 \mathrm{H}, J=8.7 \mathrm{~Hz}, \mathrm{Ar}), 7.48(\mathrm{~d}, 6 \mathrm{H}, J$ $=8.7 \mathrm{~Hz}, \mathrm{Ar}), 7.81(\mathrm{~d}, 6 \mathrm{H}, J=8.7 \mathrm{~Hz}, \mathrm{Ar}), 8.33(\mathrm{~s}, 3 \mathrm{H}$, $\mathrm{CH}=\mathrm{N}) .{ }^{13} \mathrm{C}-\mathrm{NMR}\left(\mathrm{CDCl}_{3}\right) \delta / \mathrm{ppm}: 22.75,28.91,28.98$ $\left(\mathrm{C}_{\text {aliphatic }}\right), 67.76,67.99\left(\mathrm{C}_{\text {arom }}-\mathrm{OCH}_{2}\right), 93.99,114.76$, $122.58,128.89,130.63,132.13\left(\mathrm{C}_{\text {arom }}\right), 151.35\left(\mathrm{C}_{\text {arom }}{ }^{-}\right.$ $\mathrm{N}), 118.83\left(\mathrm{C}_{\text {arom }}-\mathrm{Br}\right) 160.02(\mathrm{C}=\mathrm{N}), 160.92,161.99$ $\left(\mathrm{C}_{\text {arom }}-\mathrm{O}\right)$.

PSB-BR-6: Yield: $38 \%$ Beige precipitate. Elemental analysis: found, $\mathrm{C}$ 63.45, H 5.57, N 3.61; calculated $\left(\mathrm{C}_{63} \mathrm{H}_{66} \mathrm{~N}_{3} \mathrm{O}_{6} \mathrm{Br}_{3}\right), \mathrm{C}$ 63.01, H 5.54, N 3.50. IR (KBr) $v / \mathrm{cm}^{-1}: 2940,2866$ (C-H aliphatic), $1620(\mathrm{C}=\mathrm{N}), 1572$, 1605 (C=C), 1250 (C-O ether). ${ }^{1} \mathrm{H}-\mathrm{NMR}\left(\mathrm{CDCl}_{3}\right)$

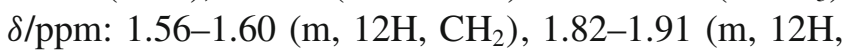
$\left.\mathrm{CH}_{2}\right), 3.95\left(\mathrm{t}, 6 \mathrm{H}, J=6.3 \mathrm{~Hz}, \mathrm{OCH}_{2}\right), 4.05(\mathrm{t}, 6 \mathrm{H}, J$ $\left.=6.3 \mathrm{~Hz}, \mathrm{OCH}_{2}\right), 6.09(\mathrm{~s}, 3 \mathrm{H}, \mathrm{Ar}), 6.98(\mathrm{~d}, 6 \mathrm{H}, J=$ $8.7 \mathrm{~Hz}, \mathrm{Ar}), 7.08$ (d, 6H, $J=8.7 \mathrm{~Hz}, \mathrm{Ar}), 7.50$ (d, 6H, $J$ $=8.7 \mathrm{~Hz}, \mathrm{Ar}), 7.83(\mathrm{~d}, 6 \mathrm{H}, J=9.0 \mathrm{~Hz}, \mathrm{Ar}), 8.36(\mathrm{~s}, 3 \mathrm{H}$, $\mathrm{CH}=\mathrm{N}) .{ }^{13} \mathrm{C}-\mathrm{NMR}\left(\mathrm{CDCl}_{3}\right) \delta / \mathrm{ppm}: 29.57,29.50,26.29$, $26.22\left(\mathrm{C}_{\text {aliphatic }}\right), 68.21,68.46\left(\mathrm{C}_{\mathrm{arom}}-\mathrm{OCH}_{2}\right), 94.16$, $115.16,123.01,130.22,131.09,132.53,\left(C_{\text {arom }}\right), 119.25$ $\left(\mathrm{C}_{\text {arom }}-\mathrm{Br}\right), 151.64\left(\mathrm{C}_{\text {arom }}-\mathrm{N}\right), 160.45(\mathrm{C}=\mathrm{N}), 161.35$, $162.47\left(\mathrm{C}_{\mathrm{arom}}-\mathrm{O}\right)$.

\subsection{Synthesis of 1,3,5-tris\{4-[(4-substituted-}

phenyl)diazenyl]phenoxy\}-n-alkyloxy benzene,

PAZ-X-n $(X=C l, B r ; n=3,4,5,6)$

The star-shaped mesogens PAZ-X-n were synthesized by employing the similar procedure as mentioned in Section 2.6.

PAZ-CL-3: Yield: 37\% Yellow precipitate. Elemental analysis: found, $\mathrm{C} 64.96, \mathrm{H} 4.82, \mathrm{~N} 8.91$; calculated $\left(\mathrm{C}_{51} \mathrm{H}_{45} \mathrm{~N}_{6} \mathrm{O}_{6} \mathrm{Cl}_{3}\right), \mathrm{C} 64.87, \mathrm{H} 4.80, \mathrm{~N} 8.90$. IR (KBr) v/cm ${ }^{-1}$ : 2952, 2873 (C-H aliphatic), 1588, $1600(\mathrm{C}=\mathrm{C}), 1473(\mathrm{~N}=\mathrm{N}), 1250$ (C-O ether). ${ }^{1} \mathrm{H}-\mathrm{NMR}$ $\left(\mathrm{CDCl}_{3}\right) \delta / \mathrm{ppm}: 2.24-2.31\left(\mathrm{~m}, 6 \mathrm{H}, \mathrm{CH}_{2}\right), 4.13(\mathrm{t}, 6 \mathrm{H}$, $\left.J=6.0 \mathrm{~Hz}, \mathrm{OCH}_{2}\right), 4.22\left(\mathrm{t}, 6 \mathrm{H}, J=6.0 \mathrm{~Hz}, \mathrm{OCH}_{2}\right)$, 
$6.13(\mathrm{~s}, 3 \mathrm{H}, \mathrm{Ar}), 7.00(\mathrm{~d}, 6 \mathrm{H}, J=9.0 \mathrm{~Hz}, \mathrm{Ar}), 7.45$ $(\mathrm{d}, 6 \mathrm{H}, J=9.0 \mathrm{~Hz}, \mathrm{Ar}), 7.81(\mathrm{~d}, 6 \mathrm{H}, J=8.5 \mathrm{~Hz}$, $\mathrm{Ar}), 7.88(\mathrm{~d}, 6 \mathrm{H}, J=9.0 \mathrm{~Hz}, \mathrm{Ar}) .{ }^{13} \mathrm{C}-\mathrm{NMR}\left(\mathrm{CDCl}_{3}\right)$

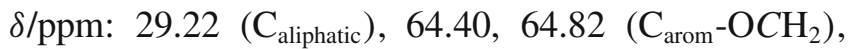
94.29, 114.80, 123.82, 124.87, $129.25\left(\mathrm{C}_{\text {arom }}\right), 136.17$ $\left(\mathrm{C}_{\text {arom }}-\mathrm{Cl}\right), 146.91,151.14\left(\mathrm{C}_{\text {arom }}-\mathrm{N}\right), 160.76,161.59$ $\left(\mathrm{C}_{\text {arom }}-\mathrm{O}\right)$.

PAZ-CL-4: Yield: $35 \%$ Yellow precipitate. Elemental analysis: found, $\mathrm{C}$ 65.73, $\mathrm{H}$ 5.25, $\mathrm{N} 8.50$; calculated $\left(\mathrm{C}_{54} \mathrm{H}_{51} \mathrm{~N}_{6} \mathrm{O}_{6} \mathrm{Cl}_{3}\right), \mathrm{C} 65.75, \mathrm{H}$ 5.21, N 8.52. IR (KBr) $v / \mathrm{cm}^{-1}: 2951,2872$ (C-H aliphatic), 1586, $1600(\mathrm{C}=\mathrm{C}), 1477(\mathrm{~N}=\mathrm{N}), 1250$ (C-O ether). ${ }^{1} \mathrm{H}-\mathrm{NMR}$

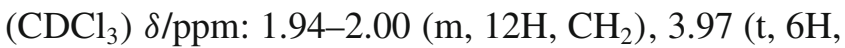
$\left.J=5.7 \mathrm{~Hz}, \mathrm{OCH}_{2}\right), 4.08\left(\mathrm{t}, 6 \mathrm{H}, J=5.7 \mathrm{~Hz}, \mathrm{OCH}_{2}\right)$, $6.05(\mathrm{~s}, 3 \mathrm{H}, \mathrm{Ar}), 6.97(\mathrm{~d}, 6 \mathrm{H}, J=9.0 \mathrm{~Hz}, \mathrm{Ar}), 7.43$ $(\mathrm{d}, 6 \mathrm{H}, J=8.7 \mathrm{~Hz}, \mathrm{Ar}), 7.80(\mathrm{~d}, 6 \mathrm{H}, J=8.7 \mathrm{~Hz}$, Ar), $7.88(\mathrm{~d}, 6 \mathrm{H}, J=9.0 \mathrm{~Hz}, \mathrm{Ar}) .{ }^{13} \mathrm{C}-\mathrm{NMR}\left(\mathrm{CDCl}_{3}\right)$

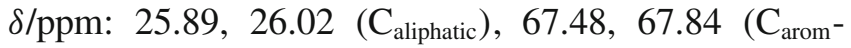
$\left.\mathrm{OCH}_{2}\right), 94.05,114.79,123.82,124.88,129.25\left(\mathrm{C}_{\text {arom }}\right)$, $136.15\left(\mathrm{C}_{\text {arom }}-\mathrm{Cl}\right), 146.83,151.17\left(\mathrm{C}_{\text {arom }}-\mathrm{N}\right), 160.86$, $161.77\left(\mathrm{C}_{\text {arom }}-\mathrm{O}\right)$.

PAZ-CL-5: Yield: 35\% Yellow precipitate. Elemental analysis: found, $\mathrm{C} 66.70, \mathrm{H}$ 5.67, $\mathrm{N} 8.21$; calculated $\left(\mathrm{C}_{57} \mathrm{H}_{57} \mathrm{~N}_{6} \mathrm{O}_{6} \mathrm{Cl}_{3}\right), \mathrm{C} 66.57, \mathrm{H}$ 5.59, N 8.17. IR (KBr) $v / \mathrm{cm}^{-1}:$ 2944, 2870 (C-H aliphatic), 1582, $1600(\mathrm{C}=\mathrm{C}), 1477(\mathrm{~N}=\mathrm{N}), 1250$ (C-O ether). ${ }^{1} \mathrm{H}-\mathrm{NMR}$

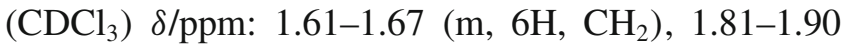
$\left(\mathrm{m}, 12 \mathrm{H}, \mathrm{CH}_{2}\right), 3.94\left(\mathrm{t}, 6 \mathrm{H}, J=6.5 \mathrm{~Hz}, \mathrm{OCH}_{2}\right), 4.04$ $\left(\mathrm{t}, 6 \mathrm{H}, J=6.5 \mathrm{~Hz}, \mathrm{OCH}_{2}\right), 6.06(\mathrm{~s}, 3 \mathrm{H}, \mathrm{Ar}), 6.97$ $(\mathrm{d}, 6 \mathrm{H}, J=8.5 \mathrm{~Hz}, \mathrm{Ar}), 7.43(\mathrm{~d}, 6 \mathrm{H}, J=8.5 \mathrm{~Hz}$, Ar), $7.80(\mathrm{~d}, 6 \mathrm{H}, J=8.5 \mathrm{~Hz}, A r), 7.87(\mathrm{~d}, 6 \mathrm{H}$, $J=9.0 \mathrm{~Hz}, \mathrm{Ar}) .{ }^{13} \mathrm{C}-\mathrm{NMR}\left(\mathrm{CDCl}_{3}\right) \delta / \mathrm{ppm}: 22.75$, $28.93,28.98\left(\mathrm{C}_{\text {aliphatic }}\right), 67.75,68.15\left(\mathrm{C}_{\text {arom }}-\mathrm{OCH}_{2}\right)$, 93.97, 114.76, 123.81, 124.88, $129.15\left(\mathrm{C}_{\text {arom }}\right), 136.12$ $\left(\mathrm{C}_{\text {arom }}-\mathrm{Cl}\right), 146.77,151.16\left(\mathrm{C}_{\text {arom }}-\mathrm{N}\right), 160.91,161.84$ $\left(\mathrm{C}_{\text {arom }}-\mathrm{O}\right)$.

PAZ-CL-6: Yield: 32\% Yellow precipitate. Elemental analysis: found, $\mathrm{C} 67.43, \mathrm{H} 5.84, \mathrm{~N} 7.89$; calculated $\left(\mathrm{C}_{60} \mathrm{H}_{63} \mathrm{~N}_{6} \mathrm{O}_{6} \mathrm{Cl}_{3}\right), \mathrm{C}$ 67.32, H 5.93, N 7.85. IR (KBr) $v / \mathrm{cm}^{-1}: 2940,2867$ (C-H aliphatic), 1583, $1600(\mathrm{C}=\mathrm{C}), 1472(\mathrm{~N}=\mathrm{N}), 1250$ (C-O ether). ${ }^{1} \mathrm{H}-\mathrm{NMR}$

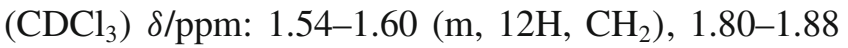
$\left(\mathrm{m}, 12 \mathrm{H}, \mathrm{CH}_{2}\right), 3.95\left(\mathrm{t}, 6 \mathrm{H}, J=6.6 \mathrm{~Hz}, \mathrm{OCH}_{2}\right), 4.06$ (t, $\left.6 \mathrm{H}, J=6.6 \mathrm{~Hz}, \mathrm{OCH}_{2}\right), 6.09(\mathrm{~s}, 3 \mathrm{H}, \mathrm{Ar}), 7.01$ $(\mathrm{d}, 6 \mathrm{H}, J=9.0 \mathrm{~Hz}, \mathrm{Ar}), 7.47(\mathrm{~d}, 6 \mathrm{H}, J=9.0 \mathrm{~Hz}$, Ar), $7.84(\mathrm{~d}, 6 \mathrm{H}, J=9.0 \mathrm{~Hz}, \mathrm{Ar}), 7.91(\mathrm{~d}, 6 \mathrm{H}, J=$ $9.0 \mathrm{~Hz}, \mathrm{Ar}) .{ }^{13} \mathrm{C}-\mathrm{NMR}\left(\mathrm{CDCl}_{3}\right) \delta / \mathrm{ppm}: 25.82,25.88$, $29.12,29.17\left(\mathrm{C}_{\text {aliphatic }}\right), 67.84,68.19\left(\mathrm{C}_{\text {arom }}-\mathrm{OCH}_{2}\right)$, 93.94, 114.78, 123.81, 124.88, $129.24\left(\mathrm{C}_{\text {arom }}\right), 136.10$ $\left(\mathrm{C}_{\text {arom }}-\mathrm{Cl}\right), 146.76,151.18\left(\mathrm{C}_{\text {arom }}-\mathrm{N}\right), 160.97,161.90$ $\left(\mathrm{C}_{\text {arom }}-\mathrm{O}\right)$.

PAZ-BR-3: Yield: 41\% Yellow precipitate. Elemental analysis: found, C 56.93, H 4.37, N 7.86; calculated
$\left(\mathrm{C}_{51} \mathrm{H}_{45} \mathrm{~N}_{6} \mathrm{O}_{6} \mathrm{Br}_{3}\right), \mathrm{C} 56.84, \mathrm{H} 4.21, \mathrm{~N}$ 7.80. IR (KBr) $v / \mathrm{cm}^{-1}:$ 2942, 2870 (C-H aliphatic), 1584-1600 (C=C), $1475(\mathrm{~N}=\mathrm{N}), 1250$ (C-O ether). ${ }^{1} \mathrm{H}-\mathrm{NMR}\left(\mathrm{CDCl}_{3}\right)$

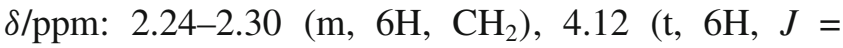
$\left.6.0 \mathrm{~Hz}, \mathrm{OCH}_{2}\right), 4.21\left(\mathrm{t}, 6 \mathrm{H}, J=6.0 \mathrm{~Hz}, \mathrm{OCH}_{2}\right)$, $6.12(\mathrm{~s}, 3 \mathrm{H}, \mathrm{Ar}), 6.99(\mathrm{~d}, 6 \mathrm{H}, J=9.0 \mathrm{~Hz}, \mathrm{Ar}), 7.60$ $(\mathrm{d}, 6 \mathrm{H}, J=8.5 \mathrm{~Hz}, \mathrm{Ar}), 7.73(\mathrm{~d}, 6 \mathrm{H}, J=8.5 \mathrm{~Hz}$, Ar), $7.88(\mathrm{~d}, 6 \mathrm{H}, J=9.0 \mathrm{~Hz}, \mathrm{Ar}) .{ }^{13} \mathrm{C}-\mathrm{NMR}\left(\mathrm{CDCl}_{3}\right)$ S/ppm: $29.12\left(\mathrm{C}_{\text {aliphatic }}\right), 64.38,64.80\left(\mathrm{C}_{\text {arom }}-\mathrm{OCH}_{2}\right)$, $94.25,114.80,124.08,124.91,132.24\left(\mathrm{C}_{\text {arom }}\right), 124.58$ $\left(\mathrm{C}_{\mathrm{arom}}-\mathrm{Br}\right), 146.90,151.32\left(\mathrm{C}_{\mathrm{arom}}-\mathrm{N}\right), 160.76,161.62$ $\left(\mathrm{C}_{\text {arom }}-\mathrm{O}\right)$.

PAZ-BR-4: Yield: 38\% Yellow precipitate. Elemental analysis: found, $\mathrm{C} 57.94, \mathrm{H} 4.55, \mathrm{~N} 7.59$; calculated $\left(\mathrm{C}_{54} \mathrm{H}_{51} \mathrm{~N}_{6} \mathrm{O}_{6} \mathrm{Br}_{3}\right), \mathrm{C} 57.92, \mathrm{H} 4.59, \mathrm{~N} 7.51$. IR (KBr) $v / \mathrm{cm}^{-1}: 2948,2872$ (C-H aliphatic), 1582, $1599(\mathrm{C}=\mathrm{C}), 1475(\mathrm{~N}=\mathrm{N}), 1251$ (C-O ether). ${ }^{1} \mathrm{H}-\mathrm{NMR}$

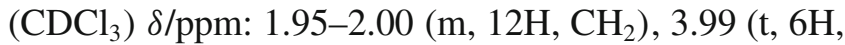
$\left.J=6.0 \mathrm{~Hz}, \mathrm{OCH}_{2}\right), 4.09\left(\mathrm{t}, 6 \mathrm{H}, J=6.0 \mathrm{~Hz}, \mathrm{OCH}_{2}\right)$, $6.07(\mathrm{~s}, 3 \mathrm{H}, \mathrm{Ar}), 6.98(\mathrm{~d}, 6 \mathrm{H}, J=9.0 \mathrm{~Hz}, \mathrm{Ar}), 7.60$ $(\mathrm{d}, 6 \mathrm{H}, J=8.5 \mathrm{~Hz}, \mathrm{Ar}), 7.74(\mathrm{~d}, 6 \mathrm{H}, J=9.0 \mathrm{~Hz}$, Ar), 7.89 (d, 6H, $J=9.0 \mathrm{~Hz}, \mathrm{Ar}) .{ }^{13} \mathrm{C}-\mathrm{NMR}\left(\mathrm{CDCl}_{3}\right)$ $\delta /$ ppm: 25.88, $26.02\left(\mathrm{C}_{\text {aliphatic }}\right), 67.47,67.83\left(\mathrm{C}_{\text {arom }}{ }^{-}\right.$ $\left.\mathrm{OCH}_{2}\right), 94.04,114.79,124.07,124.91,132.23\left(\mathrm{C}_{\text {arom }}\right)$, $124.53\left(\mathrm{C}_{\text {arom }}-\mathrm{Br}\right), 146.82,151.52\left(\mathrm{C}_{\text {arom }}-\mathrm{N}\right), 160.85$, $161.80\left(\mathrm{C}_{\text {arom }}-\mathrm{O}\right)$.

PAZ-BR-5: Yield: 38\% Yellow precipitate. Elemental analysis: found, $\mathrm{C} 59.08, \mathrm{H}$ 4.98, N 7.20; calculated $\left(\mathrm{C}_{57} \mathrm{H}_{57} \mathrm{~N}_{6} \mathrm{O}_{6} \mathrm{Br}_{3}\right), \mathrm{C} 58.93, \mathrm{H} 4.95, \mathrm{~N} 7.23$. IR $(\mathrm{KBr}) v / \mathrm{cm}^{-1}: 2940,2868$ (C-H aliphatic), 1583, $1599(\mathrm{C}=\mathrm{C}), 1475(\mathrm{~N}=\mathrm{N}), 1251$ (C-O ether). ${ }^{1} \mathrm{H}-\mathrm{NMR}$

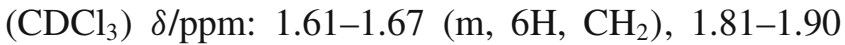
$\left(\mathrm{m}, 12 \mathrm{H}, \mathrm{CH}_{2}\right), 3.93\left(\mathrm{t}, 6 \mathrm{H}, J=6.0 \mathrm{~Hz}, \mathrm{OCH}_{2}\right), 4.04$ $\left(\mathrm{t}, 6 \mathrm{H}, J=6.5 \mathrm{~Hz}, \mathrm{OCH}_{2}\right), 6.06(\mathrm{~s}, 3 \mathrm{H}, \mathrm{Ar}), 6.97(\mathrm{~d}$, $6 \mathrm{H}, J=8.5 \mathrm{~Hz}, \mathrm{Ar}), 7.59(\mathrm{~d}, 6 \mathrm{H}, J=8.5 \mathrm{~Hz}, \mathrm{Ar})$, $7.72(\mathrm{~d}, 6 \mathrm{H}, J=9.0 \mathrm{~Hz}, \mathrm{Ar}), 7.87(\mathrm{~d}, 6 \mathrm{H}, J=9.0 \mathrm{~Hz}$, Ar). ${ }^{13} \mathrm{C}-\mathrm{NMR}\left(\mathrm{CDCl}_{3}\right) \delta /$ ppm: 22.75, 28.93, 28.98 $\left(\mathrm{C}_{\text {aliphatic }}\right), 67.75,68.15\left(\mathrm{C}_{\text {arom }}-\mathrm{OCH}_{2}\right), 93.96,114.77$, $124.07,124.91,132.24\left(\mathrm{C}_{\text {arom }}\right), 124.52\left(\mathrm{C}_{\text {arom }}-\mathrm{Br}\right)$, $146.77,151.52\left(\mathrm{C}_{\text {arom }}-\mathrm{N}\right), 160.92,161.88\left(\mathrm{C}_{\text {arom }}-\mathrm{O}\right)$.

PAZ-BR-6: Yield: 38\% Yellow precipitate. Elemental analysis: found, $\mathrm{C}$ 59.95, $\mathrm{H}$ 5.31, N 7.03; calculated $\left(\mathrm{C}_{60} \mathrm{H}_{63} \mathrm{~N}_{6} \mathrm{O}_{6} \mathrm{Br}_{3}\right)$, C 59.86, H 5.27, N 6.98. IR (KBr) $v / \mathrm{cm}^{-1}: 2941,2868$ (C-H aliphatic), 1583, $1600(\mathrm{C}=\mathrm{C}), 1473(\mathrm{~N}=\mathrm{N}), 1247$ (C-O ether). ${ }^{1} \mathrm{H}-\mathrm{NMR}$ $\left(\mathrm{CDCl}_{3}\right) \delta / \mathrm{ppm}$ : $1.51-1.58\left(\mathrm{~m}, 12 \mathrm{H}, \mathrm{CH}_{2}\right), 1.79-1.86$ $\left(\mathrm{m}, 12 \mathrm{H}, \mathrm{CH}_{2}\right), 3.93\left(\mathrm{t}, 6 \mathrm{H}, J=6.0 \mathrm{~Hz}, \mathrm{OCH}_{2}\right), 4.04$ (t, $6 \mathrm{H}, J=6.5 \mathrm{~Hz}, \mathrm{OCH}_{2}$ ), 6.07 (s, 3H, Ar), 6.99 $(\mathrm{d}, 6 \mathrm{H}, J=8.5 \mathrm{~Hz}, \mathrm{Ar}), 7.61(\mathrm{~d}, 6 \mathrm{H}, J=8.5 \mathrm{~Hz}$, Ar), $7.75(\mathrm{~d}, 6 \mathrm{H}, J=8.5 \mathrm{~Hz}, \mathrm{Ar}), 7.89(\mathrm{~d}, 6 \mathrm{H}, J=$ $8.5 \mathrm{~Hz}, \mathrm{Ar}) .{ }^{13} \mathrm{C}-\mathrm{NMR}\left(\mathrm{CDCl}_{3}\right) \delta / \mathrm{ppm}: 25.81,25.87$, $29.10,29.15\left(\mathrm{C}_{\text {aliphatic }}\right), 67.79,68.20\left(\mathrm{C}_{\text {arom }}-\mathrm{OCH}_{2}\right)$, $93.80,114.75,124.05,124.90,132.22\left(\mathrm{C}_{\text {arom }}\right), 124.49$ 
$\left(\mathrm{C}_{\text {arom }}-\mathrm{Br}\right), 146.70,151.48\left(\mathrm{C}_{\text {arom }}-\mathrm{N}\right), 160.92,161.90$ $\left(\mathrm{C}_{\text {arom }}-\mathrm{O}\right)$.

\section{Results and discussion}

\subsection{Thermal stability and optical properties for $\boldsymbol{P S B}-\boldsymbol{X}-\boldsymbol{n}$ and $\boldsymbol{P A Z}-\boldsymbol{X}-\boldsymbol{n}$}

The phase sequences, transition temperatures and associated enthalpies of the star-shaped compounds PSB$\mathbf{X}-\boldsymbol{n}$ and PAZ-X- $\boldsymbol{n}$ upon heating and cooling are tabulated in table 2. All the star-shaped mesogens exhibit liquid crystalline behaviour except for the homologues possessing the shortest alkyl spacer $(n=3)$. These compounds undergo direct isotropization on heating and crystallization on cooling, thus indicating the nonmesogenic properties. In addition, the non-mesogenic properties can also be inferred from the DSC thermograms of these homologues which display only one endotherm and exotherm upon heating-cooling cycle. The absence of mesophase is presumably due to the short flexible spacer as the short chain may tend to hinder the peripheral units from the appropriate anisotropic arrangement in forming liquid crystalline properties. ${ }^{26}$ Furthermore, shorter alkyl spacer also does not increase the polarity and polarizability of the molecules. ${ }^{37}$ Therefore, phase generation is usually less apparent especially in molecule having short alkyl chain.

From the POM investigation on series PSB-X-n, the chloro derivatives exhibit both $\mathrm{N}$ and SmC phases upon cooling. The $\mathrm{N}$ phase is identified based on the formation of nematic droplets at transition point which eventually coalesce to give a Schlieran texture (figure 2(a)). On further cooling, a transition from the $\mathrm{N}$ phase to $\mathrm{SmC}$ phase is detected prior to crystallization (figure 2(b)). The phase sequence is also supported by the DSC thermograms of PSBCL-5 as depicted in figure 3. Interestingly, PSBCL-6 is found to be enantiotropic but for PSB-CL4 and PSB-CL-5, they only show monotropic characteristic where mesophase is only observed during the cooling cycle. As for PSB-BR-n which contain para-substituted bromo atom, focal conic fanshaped texture of SmA phase is observed when cooling PSB-BR-5 and PSB-BR-6 from the isotropic state except for member with butyl spacer $(n=4)$. Figure 4 shows the optical photomicrograph of PSBBR-5 exhibiting the SmA fan-shaped texture. Among the bromo-substituted derivatives, PSB-BR-5 is found to be monotropic (figure 5) while PSB-BR-6 is enantiotropic. Only smectic phase is observed for PSB-BR$\boldsymbol{n}$ and this can be attributed to the presence of highly polarizable bromine atom at the terminal which can enhance the overall intermolecular attraction. ${ }^{38}$ In short, series PSB-X- $\boldsymbol{n}$ reveals that star-shaped mesogens possessing butyl $(n=4)$ and pentyl $(n=5)$ spacers show only monotropic behaviour while the member possessing hexyl spacer $(n=6)$ exhibit enantiotropic properties. In addition, across the series of PSB-X- $\boldsymbol{n}$, the clearing temperatures show a regular dependence on the number of methylene units, $n$ in the spacer whereby the temperature decreases with the spacer length. For instance, the highest clearing point is recorded for PSBCL-3, whilst PSB-CL-6 possesses the lowest clearing temperature. This phenomenon can be explained by the dilution effect of the peripheral mesogenic units owing to the longer aliphatic chain of the spacer which eventually increases the flexibility of the star-shaped molecule. ${ }^{39}$

In order to establish the effect of spacer length towards the mesomorphic properties, the dependence of phase transition temperatures of these homologous series PSB-X- $\boldsymbol{n}$ on the number of methylene units, $n$ in the flexible alkyl spacers is plotted (figure 6). The commonly observed zigzag pattern or also known as the odd-even effect is hardly seen on the isotropicmesophase temperature for series PSB-X-n. Instead, the plot clearly shows that in PSB-X-n, homologues with even-parity spacer exhibit higher mesophase stability compare to the odd-member star-shaped mesogens. Besides, one distinct difference between both series is that in order for PSB-CL- $\boldsymbol{n}$ to show mesophase, the number of methylene unit $(n)$ must be at least 4, but for the bromo-derivatives PSB-BR-n, the $n$ must be at least 5. By comparing PSB-CL-4 and PSBBR-4, the chloro derivative is found to be mesogenic but PSB-BR-4 does not show liquid crystalline state. This observation can be resulted from the influence of different terminal atom in terms of their size. Thus, the effect of terminal end group is presumably to cause such alternation as the radius for bromine atom is considered larger than chlorine and thus may tend to disrupt the lamellar packing of the molecule. ${ }^{40}$ As a result, PSBBR-4 does not exhibit liquid crystalline properties due to the increased substituent size.

For the azo series PAZ-X-n, star-shaped mesogens with the chloro substituent show both $\mathrm{N}$ and SmA phase in their liquid crystalline state when viewed under a polarized optical microscope. For instance, homologues possessing the even-parity spacers PAZ-CL-4 and PAZ-CL-6 exhibit both N and SmA phases on cooling. However, the butyl analogue PAZ-CL-4 is found to be monotropic while PAZ-CL-6 is enantiotropic in nature. While for PAZ-CL-5 which is comprised of odd-parity spacer, it exhibits enantiotropic SmA 
Table 2. Phase transition temperatures $\left({ }^{\circ} \mathrm{C}\right)$ and associated enthalpy changes $\left(\mathrm{kJ} \mathrm{mol}^{-1}\right)$ for compounds PSB-X-n and PAZ-X-n upon heating and cooling cycles.

\begin{tabular}{|c|c|}
\hline Compound & Transition temperatures, ${ }^{\circ} \mathrm{C}\left(\Delta \mathrm{H}, \mathrm{kJ} \mathrm{mol}^{-1}\right)$ \\
\hline PSB-CL-3 & $\begin{array}{l}\mathrm{Cr} 139.4(65.1) \mathrm{I} \\
\mathrm{I} 70.6(7.9) \mathrm{Cr}\end{array}$ \\
\hline PSB-CL-4 & $\begin{array}{l}\mathrm{Cr} 129.8 \text { (59.8) I } \\
\mathrm{I} 103.7(1.9) \mathrm{N} 78.9 \text { (1.7) } \mathrm{SmC} 72.2 \text { (4.6) } \mathrm{Cr}\end{array}$ \\
\hline PSB-CL-5 & $\begin{array}{l}\mathrm{Cr}_{1} 93.7(9.1) \mathrm{Cr}_{2} 120.3(70.7) \mathrm{I} \\
I 98.2(1.4) N 78.8(0.8) \mathrm{SmC} 66.5 \text { (3.1) Cr }\end{array}$ \\
\hline PSB-CL-6 & $\begin{array}{l}\mathrm{Cr}_{1} 82.3(20.5) \mathrm{Cr}_{2} 101.2(6.8) \mathrm{N} 117.6(2.5) \mathrm{I} \\
I 101.4(1.9) N 81.3(0.6) \mathrm{SmC} 58.4(2.7) \mathrm{Cr}\end{array}$ \\
\hline PSB-BR-3 & $\begin{array}{l}\mathrm{Cr}_{1} 153.2(46.9) \mathrm{Cr}_{2} 168.8(34.1) \mathrm{I} \\
\text { I } 113.6(7.4) C r_{1} 97.5(13.8) C r_{2}\end{array}$ \\
\hline PSB-BR-4 & $\begin{array}{l}\mathrm{Cr} 153.7(14.5) \mathrm{I} \\
\mathrm{I} 109.4(7.8) \mathrm{Cr}\end{array}$ \\
\hline PSB-BR-5 & $\begin{array}{l}\mathrm{Cr} 145.0 \text { (71.4) I } \\
\text { I } 115.3(9.3) \mathrm{SmA} 106.8(7.0) \mathrm{Cr}\end{array}$ \\
\hline PSB-BR-6 & $\begin{array}{l}\mathrm{Cr}_{1} 67.2(16.8) \mathrm{Cr}_{2} 96.8(5.9) \mathrm{SmA} 108.1(3.0) \mathrm{I} \\
\text { I 106.7(3.9) SmA } 90.0(6.2) \mathrm{Cr}\end{array}$ \\
\hline PAZ-CL-3 & $\begin{array}{l}\mathrm{Cr}_{1} 174.5(56.2) \mathrm{I} \\
I 98.1(41.7) \mathrm{Cr}\end{array}$ \\
\hline PAZ-CL-4 & $\begin{array}{l}\mathrm{Cr} 145.7 \text { (95.2) I } \\
\text { I } 110.4(1.3) N 107.2 \text { (10.7) SmA } 94.8 \text { (34.9) Cr }\end{array}$ \\
\hline PAZ-CL-5 & $\begin{array}{l}\text { Cr } 110.0 \text { (3.7) SmA } 142.4(75.9) \mathrm{I} \\
I 120.2 \text { (12.9) SmA } 75.3(29.4) \mathrm{Cr}\end{array}$ \\
\hline PAZ-CL-6 & $\begin{array}{l}\mathrm{Cr} 76.8(1.2) \mathrm{SmA} 104.3 \text { (40.0) } 112.5 \text { (32.1) I } \\
\text { I 111.2(4.3) N } 108.7 \text { (4.8) SmA } 55.3 \text { (1.4) Cr B }\end{array}$ \\
\hline PAZ-BR-3 & $\begin{array}{l}\mathrm{Cr}_{1} 166.8(15.8) \mathrm{Cr}_{2} 181.7(54.5) \mathrm{I} \\
\text { I } 116.3(48.3) \mathrm{Cr}\end{array}$ \\
\hline PAZ-BR-4 & $\begin{array}{l}\mathrm{Cr} 154.0(71.44) \mathrm{I} \\
\text { I } 122.3 \text { (13.6) SmA } 97.6 \text { (15.0) Cr }\end{array}$ \\
\hline PAZ-BR-5 & $\begin{array}{l}\mathrm{Cr}_{1} 101.2 \text { (23.1) } \mathrm{Cr}_{2} 137.4 \text { (11.2) SmA } 152.2 \text { (76.8) I } \\
\text { I 135.1 (17.9) SmA 98.6(3.6) SmB } 94.3 \text { (6.6) Cr }\end{array}$ \\
\hline PAZ-BR-6 & $\begin{array}{l}\mathrm{Cr} 125.1(58.3) \mathrm{SmA} 133.7(33.7) \mathrm{I} \\
\text { I } 130.2 \text { (15.8) SmA } 88.0(1.7) \mathrm{Cr} B\end{array}$ \\
\hline
\end{tabular}

Cr B, Soft crystal B phase; Cr, Crystal; SmA, smectic A; SmB, smectic $\mathrm{B}$; SmC, smectic C; N, nematic; I, isotropic *Italic denotes cooling scan

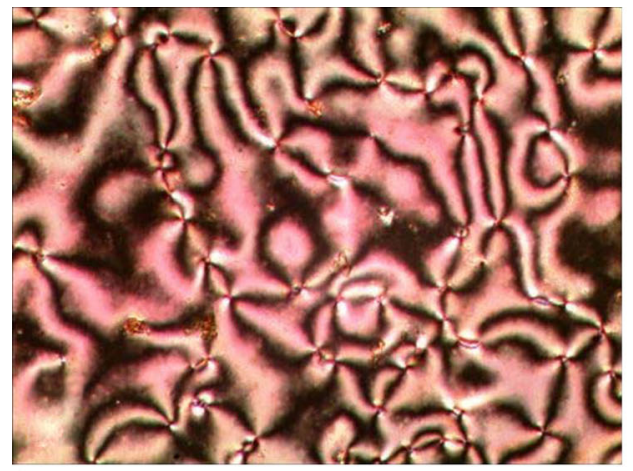

(a)

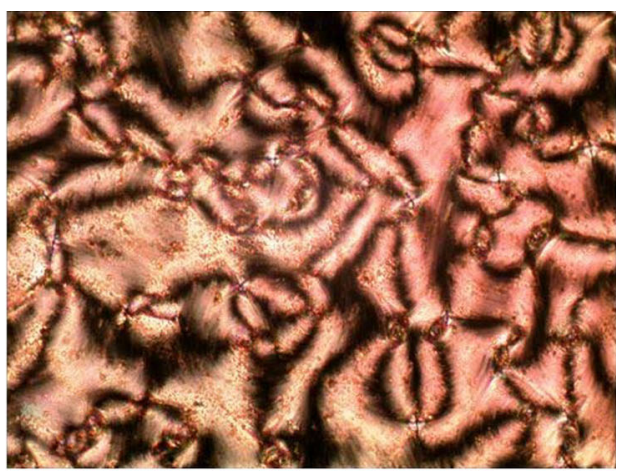

(b)

Figure 2. Optical photomicrographs of PSB-CL-5 taken during the cooling cycle. Upon cooling from the isotropic, (a) Schlieran texture two- and four-fold brushes of $\mathrm{N}$ phase appeared followed by the (b) SmC phase. 


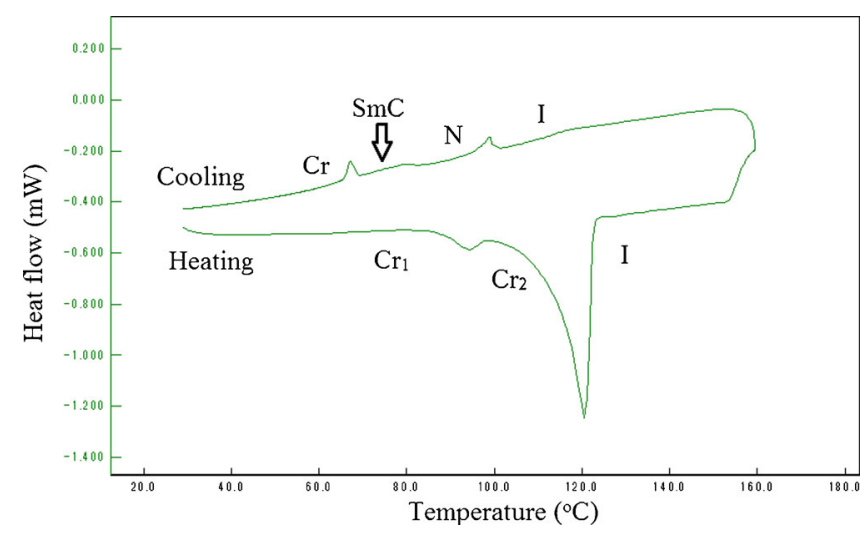

Figure 3. DSC traces of PSB-CL-5 on heating and cooling cycles at the rate of $\pm 5^{\circ} \mathrm{C}$.

phase only. A representative optical photomicrograph of PAZ-CL-4 showing the Schlieran texture of N phase and the fan-shaped texture of SmA phase are depicted in figure 7. In the series of PAZ-BR-n, the formation of mesophase is unfavourable for the short spacer, but when the number of methylene units of the spacer reaches $n=4$, mesophase is detected. The formation of batonets that coalesce to form the fan-shaped texture is the main feature of SmA phase. DSC thermograms also confirm the monotropic feature of this mesogen. POM observation on PAZ-BR-5 reveals an additional transition at lower temperature identified as $\mathrm{SmB}$ phase, which is assigned based on the appearance of transient transition bars across the phase boundary (figure 8). ${ }^{41}$ The transformation of textural feature from SmA to SmB phase only involve very subtle changes in the texture, thus this often makes the transition between these two phases difficult to be detected by POM. Thus, the existence of $\mathrm{SmB}$ phase following the SmA phase

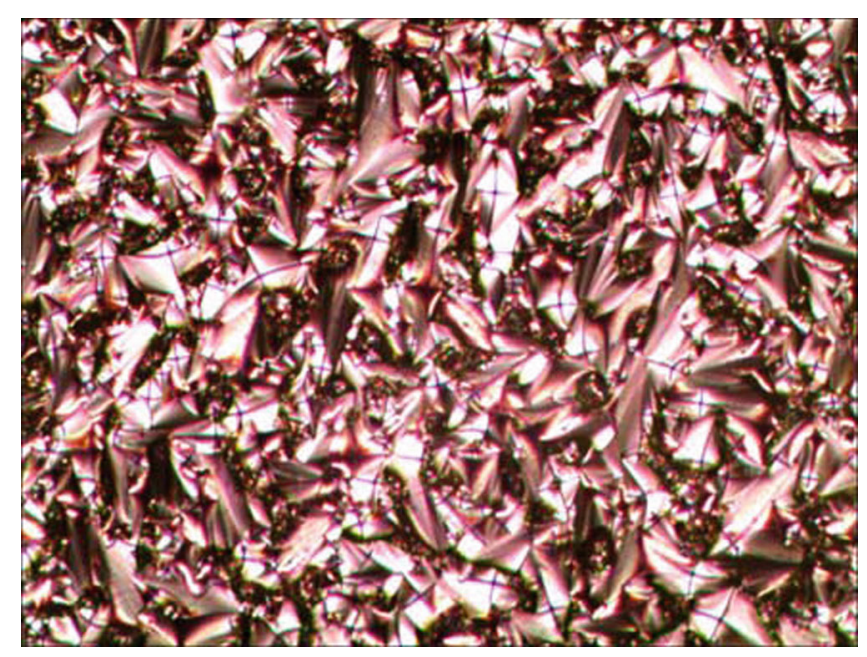

Figure 4. Optical photomicrograph of PSB-BR-5 exhibiting the focal conic fan-shaped texture of SmA phase.

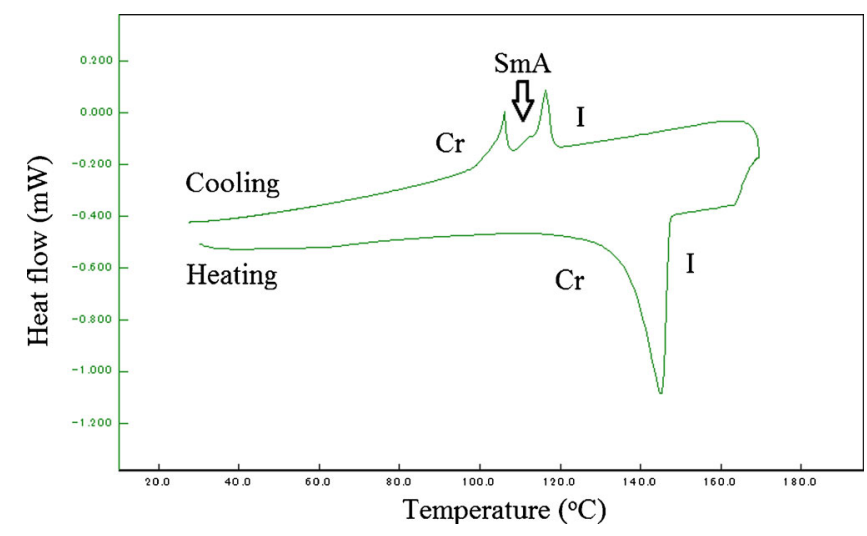

Figure 5. DSC trace of PSB-BR-5 on heating and cooling cycles at the rate of $\pm 5^{\circ} \mathrm{C}$.

in PAZ-BR-5 is further substantiated from the DSC thermograms (figure 9) whereby three phase transitions, I-SmA, SmA-SmB and SmB-Cr are detected in the cooling run.

Apart from that, star-shaped azo mesogens with hexyl spacer (PAZ-CL-6 and PAZ-BR-6) are found to exhibit an interesting feature as compared to others whereby full recrystallization from the SmA phase upon cooling to room temperature is not observed. However, from the DSC analysis, a small transition accompanied by low enthalpy value is detected at lower temperature. This phenomenon can be attributed to the emergence of additional phase transition upon cooling compounds PAZ-CL-6 and PAZ-BR-6. Hence, it is presumed that the compounds do not crystallize but transform into a soft crystal state which can be identified as soft crystal B phase. In general, soft crystalline phase cannot be distinguished unambiguously by polarizing microscope alone but require additional DSC technique to substantiate the phase transition. At room temperature, the non-crystalline texture which closely resembles the fan-shaped texture stays firm and unchanged even when mechanical stress is induced. For example, PAZ-BR-6 shows a fan-shaped texture of SmA phase at high temperature; however, this feature retained upon cooling into the soft crystal phase. From the observation through microscope, a change in birefringence is observed during the transition to soft crystal B phase. ${ }^{42}$ The DSC thermograms of PAZBR-6 showing the transition to soft crystalline state is depicted in figure 10. Therefore, PAZ-CL-6 and PAZBR-6 do not crystallize but tend to solidify into a soft crystal B phase. By referring to figure 11, the smectic phase range for PAZ-CL- $\boldsymbol{n}$ and PAZ-BR- $\boldsymbol{n}$ apparently increase upon elongation of spacer length. This is because the long alkyl chain resulting from the additional methylene units enables more flexibility of the peripheral units being attracted and aligned in parallel 


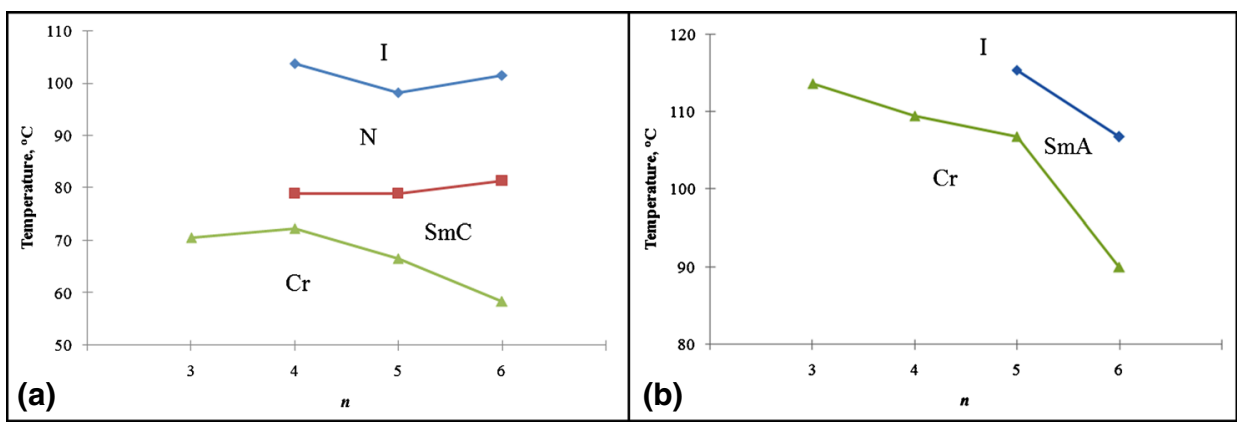

Figure 6. Mesomorphic behaviour as a function of the number of methylene unit $(n)$ in the spacer for (a) PSB-CL- $\boldsymbol{n}$ and (b) PSB-BR- $\boldsymbol{n}$.

order. This in turn facilitates the lamellar packing of the star-shaped molecules and eventually causes an increment in the smectic phase stability. ${ }^{43}$ Regardless of which terminal substituent, PAZ-X- $\boldsymbol{n}$ exhibit similar trend in the isotropic-mesophase temperature. As the spacer chain increases from $n=3$ to 5 , both homologues show a gradual increment in the I-SmA transition temperature. However, as the spacer reaches a certain length $(n=6)$, the I-SmA transition temperature become lower. Furthermore, the odd-even effect on the mesomorphic properties is also not noticeable in the azobenzene star-shaped series. Overall, the results obtained for both PSB-X- $\boldsymbol{n}$ and PAZ-X- $\boldsymbol{n}$ indicate that the length of the flexible spacer greatly affect the formation of thermotropic mesophases in this star-shaped system.

\subsection{Structure-mesomorphic property relationships:} Comparative study and influence of linking group between star-shaped mesogens PSB-X-n and PAZ-X-n

Relationship between the molecular architecture and the liquid crystalline behaviour is of fundamental importance in the field of liquid crystals study. Hence, the phase transition temperatures and the nature of mesophases exhibited by PSB-X- $\boldsymbol{n}$ and PAZ-X- $\boldsymbol{n}$ are compared in order to see the relative effect of $-\mathrm{CH}=\mathrm{N}-$ and $-\mathrm{N}=\mathrm{N}-$ linkages toward the mesomorphism of the present star-shaped mesogens. From the comparative study, both linkages are found to exhibit similar fashion as they are inclined to mesomorphism. This can be due to the even number of atoms in their structure which can eventually enhance molecular linearity, hence promoting the formation of liquid crystalline properties. ${ }^{43,44}$ By comparing the Schiff base and azobenzene group to those having odd number of atoms such as chalcone $(-\mathrm{CH}=\mathrm{CH}-\mathrm{CO}-)$, the latter is known to be less conducive to mesomorphism based on the literature. This chalcone linkage hardly exhibit any liquid crystalline behaviour because of their non-linearity and angular strain of such group resulting from the angle of keto group. ${ }^{37}$ In short, linking units which have even number of atoms like Schiff base and azo groups own great ability in altering the linearity and rigidity of the molecule since they restrict the freedom of rotation. ${ }^{45}$ Thus, they are recognized as a very useful structural component in increasing molecular

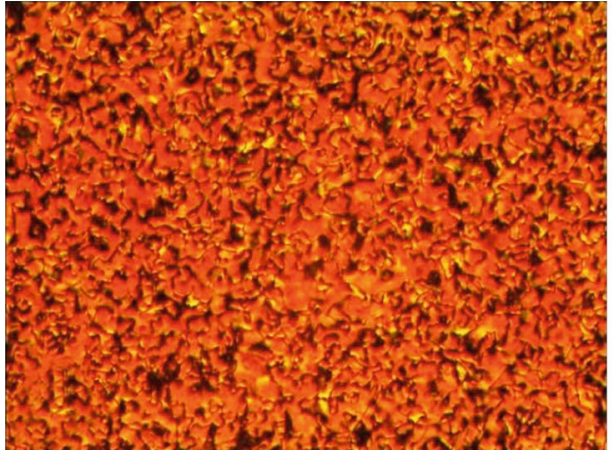

(a)

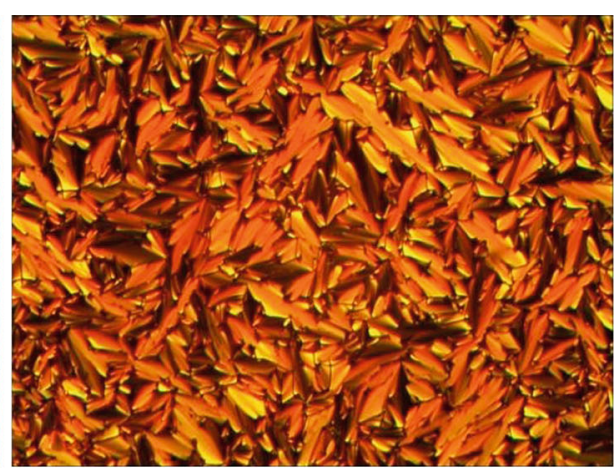

(b)

Figure 7. Optical photomicrographs of PAZ-CL-4: (a) The formation of Schlieran texture of $\mathrm{N}$ phase upon cooling and followed by the (b) fan-shaped texture characteristic of SmA phase. 


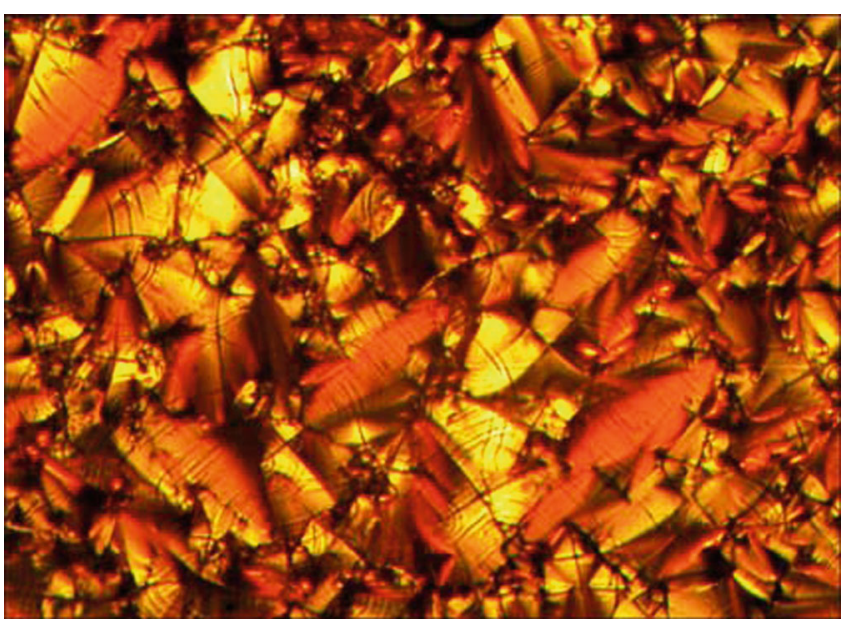

Figure 8. Optical photomicrograph of PAZ-BR-5 upon cooling showing the appearance of transition bars across the fan-shaped during the SmA to SmB phase transition.

anisotropy leading to the formation of liquid crystalline properties.

Besides, there is a common feature observed in both series. Since the introduction of halogen atom as the terminal substituent will provide the star-shaped molecules with high polarity and terminal attraction, the star-shaped compounds bearing either terminal chloro or bromo substituent are found to exhibit mesophase. By referring to table 2, all the chloro-substituted starshaped mesogens regardless of linking group and spacer chain show both nematic and smectic phases while for the bromo-substituted star-shaped mesogens, only smectic phase is detected. Therefore, the presence of 4-bromo derivatives is capable of enhancing higher tendency of smectic order relative to the 4-chlorosubstituted compounds. This finding is also in agreement with the order of decreasing smectic thermal stability of the compounds in terms of the terminal

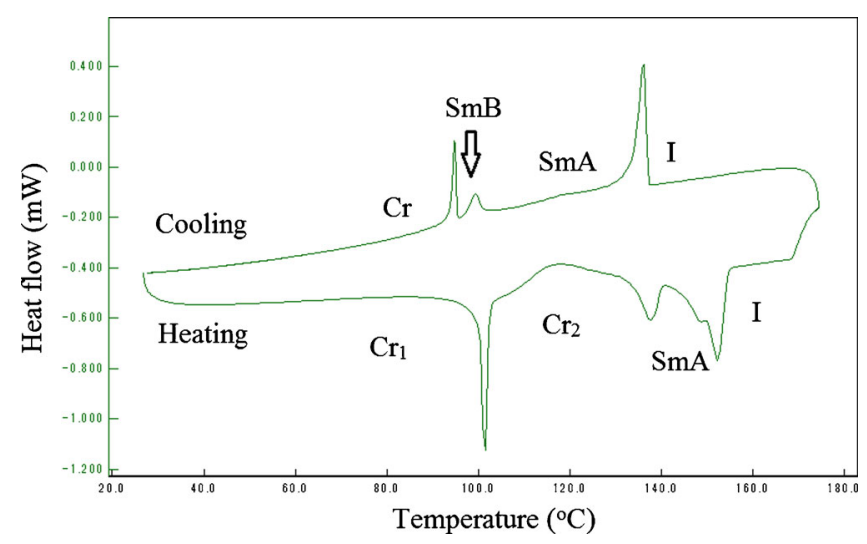

Figure 9. DSC trace of PAZ-BR-5 on heating and cooling cycles at the rate of $\pm 5^{\circ} \mathrm{C}$. substituent reported by Gray. ${ }^{46}$ One significant advantage of the azobenzene-based star-shaped liquid crystals over the Schiff base counterparts is that the former are thermally more stable than the latter. For example, the Cr-I transition temperature for PSB-CL-4 is $129.8^{\circ} \mathrm{C}$ but the azo counterpart PAZ-CL-4 is higher which is $145.7^{\circ} \mathrm{C}$. This behaviour is also observed in the unconventional V-shaped mesogens documented by Prasad. ${ }^{47}$ Furthermore, higher smecitc phase stability of the star-shaped mesogens can be achieved in the series which incorporates azobenzene as the peripheral units. The $\Delta_{\mathrm{SmA}}$ is found to be $8.5^{\circ} \mathrm{C}$ for PSBBR-5, but for PAZ-BR-5, the $\Delta_{\mathrm{SmA}}$ is higher $\left(36.5^{\circ} \mathrm{C}\right)$. Therefore, PAZ-X- $\boldsymbol{n}$ tend to favour the smectic order over the nematic which is fully compatible with the molecular arrangement resulting from the side-by-side organization of the azobenzene peripheral units which lies parallel with each other, thus stabilize the layering of the smectic phase. Through self-assembly, the symmetrical star-shaped mesogens is capable of folding an anisotropic shape to give rise to the appearance of calamitic liquid crystalline order (nematic and smectic) instead of discotic phase because the alkyl spacer between the linearly aromatic peripherals and the benzene core is flexible enough to allow the peripherals to rotate freely. ${ }^{48} \mathrm{~A}$ similar observation has also been reported for three-armed star-shaped mesogens which

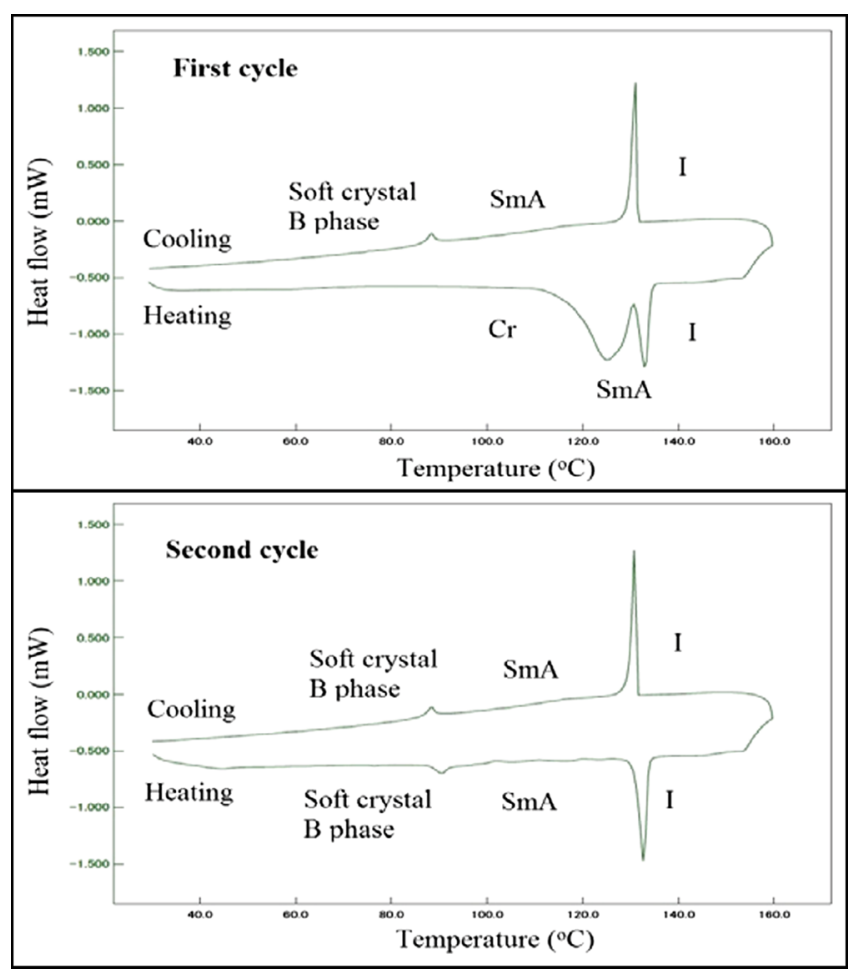

Figure 10. DSC traces of PAZ-BR-6 upon heating and cooling in the first and second cycle. 


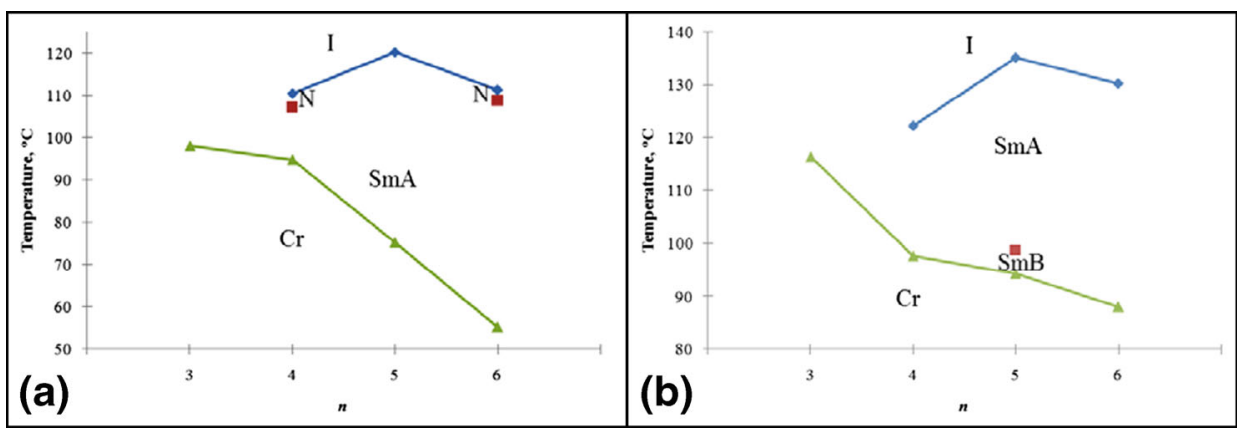

Figure 11. Mesomorphic behaviour as a function of the number of methylene unit $(n)$ in the spacer for (a) PAZ-CL- $\boldsymbol{n}$ and (b) PAZ-BR- $\boldsymbol{n}$.

incorporate three rod-shaped azobenzene molecular sub-units joining to the 2,4,6-positions of the triazine nucleus. ${ }^{30,49}$ Therefore, variation of the peripheral units in both series made it possible to observe the effects of structural changes on the star-shaped mesomorphic system which has not been studied previously.

\subsection{Spectroscopic analysis}

3.3a FT-IR spectroscopy: FT-IR discussion on the three-armed star-shaped compounds will be based on the representative homologues of PSB-CL-3 and PAZ-

BR-5. The absence of the -OH stretching signal in both spectra indicates that the alkylation reaction between the intermediate and phloroglucinol has taken place. In the FT-IR spectrum of PSB-CL-3, two distinct sharp absorption bands located at the frequency range of $2870-2916 \mathrm{~cm}^{-1}$ can be attributed to the C-H aliphatic stretching. As for the azo derivative PAZ-BR-5, same type of stretching frequency attributed to the aliphatic methylene chain is observed at the region of 2868$2940 \mathrm{~cm}^{-1}$. On the other hand, the existence of strong absorption bands at the frequency range of 1571$1602 \mathrm{~cm}^{-1}$ in both spectra of PSB-CL-3 and PAZ-BR5 can be ascribed to the $\mathrm{C}=\mathrm{C}$ of phloroglucinol benzene core and the aromatic fragments of the peripheral units. In addition, the presence of $\mathrm{C}-\mathrm{O}$ ether linkage in both star-shaped compounds gives rise to an intense absorption band at $1250-1251 \mathrm{~cm}^{-1}$ which indicates the occurrence of alkylation. The medium absorption band observed in the FT-IR spectrum of PSBCL-3 at $1619 \mathrm{~cm}^{-1}$ can be assigned to the stretching of azomethine group which connects the two aromatic rings of 4-chloroaniline and 4-hydroxybenzaldehyde. While for PAZ-BR-5, the stretching of $\mathrm{N}=\mathrm{N}$ absorption band can be found at $1475 \mathrm{~cm}^{-1}$. This value is also in agreement with the absorption bands of azo stretching for 2,2'-(4-(alkyloxy)-1,3-phenylene)bis(1(4-substituted phenyl)diazene) as highlighted in the literature. ${ }^{50}$ Overall, it can be concluded that the absorption bands associated with the selected functional groups are consistent in terms of frequencies.

\section{Conclusion}

In this article, we have presented the synthesis and characterization as well as the mesomorphic behaviour of two star-shaped homologues series, 1,3,5-tris \{4-[(4substituted-phenylimino)methyl]phenoxy $\}$-n-alkyloxy benzene (PSB-X-n) which comprised of Schiff base peripheral units and 1,3,5-tris\{4-[(4-substitutedphenyl)diazenyl]phenoxy - -alkyloxy benzene (PAZ$\mathbf{X}-\boldsymbol{n})$ which comprised of azobenzene moieties as the mesogenic side-chain. Star-shaped compounds with short spacer are non-mesogenic. N, SmA and SmC phases are detected in PSB-X-n while PAZ-X-n exhibit both N, SmA and SmB phases. An additional feature found in the series of PAZ-X- $\boldsymbol{n}$ is that the hexyl homologues tend to form soft crystalline phase on cooling. The molecular structures of peripheral rod-shaped Schiff base and azobenzene fragment accompanied with the non-polar aliphatic spacer determine the thermal stability as well as the structure of mesophase formed by the star-shaped mesogens. In short, the liquid crystalline properties should be driven by the rod-like units and the benzene core act only as a linking unit interconnecting the rods. The Schiff base and azobenzene groups are able to increase the linearity and polarizability of the molecule, thus making it possible in the generation of mesomorphism.

\section{Acknowledgements}

The author G-YY would like to thank Universiti Sains Malaysia for providing the research funding through RU Grant No 203/PKIMIA/6711265. Y-HO would like to thank the Ministry of Higher Education Malaysia (MOHE) for the scholarship under the scheme MyBrain15. 


\section{References}

1. Achalkumar A S, Hiremath U S, Shankar Rao D S and Yelamaggad C V 2011 Liq. Cryst. 381563

2. Gemming S, Popov I and Lehmann M 2007 Philos. Mag. Lett. 87883

3. Tschierske C 1998 J. Mater. Chem. 81485

4. Yoshizawa A 2008 J. Mater. Chem. 182877

5. Tschierske C 2001 Annu. Rep. Prog. Chem. Sect. C 97 191

6. Tian M, Zhang B Y, Cong Y H, He X Z, Chu H S and Zhang X Y 2010 Liq. Cryst. 371373

7. Tian M, Zhang B Y, Cong Y H, Zhang N and Yao D S 2009 J. Mol. Struct. 92339

8. Yao D S, Zhang B Y, Li Y H and Xiao X Q 2004 Tetrahedron Lett. 458953

9. Donaldson T, Henderson P A, Achard M F and Imrie C T 2011 Liq. Cryst. 381331

10. Henderson P A and Imrie C T 2005 Liq. Cryst. 32 1531

11. Yeap G Y, Hng T C, Mahmood W A K, Gorecka E, Takeuchi D and Osakada K 2009 Mol. Cryst. Liq. Cryst. 506109

12. Imrie C T, Henderson P A and Yeap G Y 2009 Liq. Cryst. 36755

13. Nishiyama I, Yamamoto J, Goodby J W and Yokoyama H 2003 J. Mater. Chem. 132429

14. Umadevi S and Sadashiva B K 2005 Liq. Cryst. 321233

15. Yelamaggad C V, Shashikala I, Shankar Rao D S and Prasad S K 2004 Liq. Cryst. 311027

16. Prasad V 2001 Liq. Cryst. 28145

17. Wang H, Zheng Z and Shen D 2012 Liq. Cryst. 3999

18. Balamurugan S and Kannan P 2009 J. Mol. Struct. 934 44

19. Jung B M, Huang Y D and Chang J Y 2010 Liq. Cryst. 3785

20. Abe Y, Nakazima N, Tanase T, Katano S, Mukai H and Ohta K 2007 Mol. Cryst. Liq. Cryst. 466129

21. Chae H W, Kadkin O N and Choi M G 2009 Liq. Cryst. 3653

22. Rezvani Z, Divband B, Abbasi A R and Nejati K 2006 Polyhedron 251915

23. Lu H, Wang J and Song X 2011 Mol. Cryst. Liq. Cryst. 53793

24. Lee J H, Han M J, Hwang S H, Jang I, Lee S J, Yoo S H, Jho J Y and Park S Y 2005 Tetrahedron Lett. 46 7143

25. Lee S J, You M K, Lee S W, Lee J, Lee J H and Jho J Y 2011 Liq. Cryst. 381289

26. Lehmann M 2012 Top. Curr. Chem. 318193

27. Lehmann M 2009 Chem. Eur. J 153638
28. Yao D S, Zhang B Y, Zhang W W and Tian M $2008 \mathrm{~J}$. Mol. Struct. $\mathbf{8 8 1} 83$

29. Zhang B Y, Yao D S, Meng F B and Li Y H 2005 J. Mol. Struct. 741135

30. Salisu A A and Kogo A A 2010 Bayero J. Pure Appl. Sci. 354

31. Meier H, Lehmann M, Holst H C and Schwöppe D 2004 Tetrahedron 606881

32. Lehmann M, Gearba R I, Ivanov D A and Koch M H J 2004 Mol. Cryst. Liq. Cryst. 411397

33. Stackhouse P J, Wilson A, Lacey D and Hird M 2010 Liq. Cryst. 371191

34. Pfeuffer T, Hanft D and Strohriegl P 2002 Liq. Cryst. 29 1555

35. Yeap G Y, Hng T C, Yeap S Y, Gorecka E, Ito M M, Ueno K, Okamoto M, Mahmood W A K and Imrie C T 2009 Liq. Cryst. 361431

36. García T, Larios-López L, Rodîguez-González R J, Martínez-Ponce G, Solano C and Navarro-Radîhuez D 2012 Polymer 532049

37. Thaker B T and Kanojiya J B 2011 Mol. Cryst. Liq. Cryst. 542 84/[606].

38. Yeap G Y, Lee H C, Mahmood W A K, Imrie C T, Takeuchi D and Osakada K 2011 Ph. Trans. 8429

39. Yeap G Y, Al-Taifi E A, Ong C H, Mahmood W A K, Takeuchi D and Ito M M 2012 Ph. Trans. 85483

40. Filippov S K, Kolomiets I P, Sokolova O S, Antonov E A, Zorin I M and Bilibin A Y 1998 Liq. Cryst. 24787

41. Goodby J W and Gray G W 1979 J. Phys. 4363

42. Dierking I 2003 Textures of liquid crystals (Weinheim, Germany: Wiley-VCH) p. 141.

43. Collings P J and Hird M 1998 Introduction to liquid crystals: Chemistry and physics (London, UK: Taylor \& Francis Ltd)

44. Singh S and Dunmur D A 2002 Liquid crystals: Fundamentals (London, UK: World Scientific Publishing Co Pvt Ltd) p. 103.

45. Demus D, Goodby J W, Gray G W, Spiess H W and Vill V 1998 Handbook of liquid crystals Vol 3: High molecular liquid crystals (Weinheim, Germany: Wiley-VCH) p. 40.

46. Gray G W 1962 Molecular structure and the properties of liquid crystals (London, UK: Academic)

47. Prasad V 2001 Liq. Cryst. 28145

48. Attard G S, Douglass A G, Imrie C T and Taylor L 1992 Liq. Cryst. 11779

49. Goldmann D, Janietz D, Schmidt C and Wendorff J H 1998 Liq. Cryst. 25711

50. Yeap G Y, Ong C H, Takeuchi D, Kakeya M, Osakada K, Mahmood W A K, Atsuko O and Vill V 2008 J. Mol. Struct. $\mathbf{8 8 2} 1$ 